\title{
Is it time to RE-AIM? A systematic review of economic empowerment as HIV prevention intervention for adolescent girls and young women in sub-Saharan Africa using the RE- AIM framework
}

Juliet Iwelunmor 1*, Ucheoma Nwaozuru', Chisom Obiezu-Umeh', Florida Uzoaru', John Ehiri², Jami Curley', Oliver Ezechi ${ }^{3}$, Collins Airhihenbuwa ${ }^{4}$ and Fred Ssewamala ${ }^{5}$

\begin{abstract}
Background: Economic empowerment (EE) HIV prevention programs for adolescent girls and young women (AGM) in subSaharan Africa are gaining traction as effective strategies to reduce HIV risk and vulnerabilities among this population. While intervention effectiveness is critical, there are numerous factors beyond effectiveness that shape an intervention's impact. The objective of this systematic review was to assess the reporting of implementation outcomes of EE HIV prevention programs for AGYW in SSA, as conceptualized in the RE-AIM (reach, efficacy/effectiveness, adoption, implementation, and maintenance) framework.

Methods: We searched PubMed, Ovid/MEDLINE, Science Direct, Ebscohost, PsyclNFO, Scopus, and Web of Science for EE HIV interventions for AGYW in SSA. Study selection and data extraction were conducted according to the PRISMA (Preferred Reporting Items for Systematic Reviews and Meta-analyses) guidelines. Two researchers coded each article using a validated RE-AIM data extraction tool and independently extracted information from each article. The reporting of RE-AIM dimensions were summarized and synthesized across included interventions.

Results: A total of 25 unique interventions (reported in 45 articles) met the predefined eligibility criteria. Efficacy/effectiveness 19(74.4\%) was the highest reported RE-AlM dimension, followed by adoption 17(67.2\%), reach 16(64.0\%), implementation 9(38.0\%), and maintenance 7(26.4\%). Most interventions reported on RE-AIM components such as sample size 25(100.0\%), intervention location 24(96.0\%), and measures and results for at least one follow-up 24(96.0\%). Few reported on RE-AIM components such as characteristics of non-participants 8(32.0\%), implementation costs 3(12.0\%), and intervention fidelity $0(0.0 \%)$.
\end{abstract}

* Correspondence: Julietiwelunmor@sluedu

${ }^{1}$ College for Public Health and Social Justice, Saint Louis University, Salus Center, 3545 Lafayette Avenue, Saint Louis, MO 63104, USA

Full list of author information is available at the end of the article

C C The Author(s). 2020 Open Access This article is licensed under a Creative Commons Attribution 4.0 International License, which permits use, sharing, adaptation, distribution and reproduction in any medium or format, as long as you give appropriate credit to the original author(s) and the source, provide a link to the Creative Commons licence, and indicate if changes were made. The images or other third party material in this article are included in the article's Creative Commons licence, unless indicated otherwise in a credit line to the material. If material is not included in the article's Creative Commons licence and your intended use is not permitted by statutory regulation or exceeds the permitted use, you will need to obtain permission directly from the copyright holder. To view a copy of this licence, visit http://creativecommons.org/licenses/by/4.0/ The Creative Commons Public Domain Dedication waiver (http://creativecommons.org/publicdomain/zero/1.0/) applies to the data made available in this article, unless otherwise stated in a credit line to the data. 


\section{Contributions to the literature}

- This study addresses an important gap in knowledge given the paucity of evidence regarding the extent to which existing economic empowerment as HIV prevention interventions for AGYW in SSA report on components of the RE-AIM framework.

- The RE-AIM framework is a useful evaluation framework for assessing scale-up, dissemination, or implementation of economic empowerment as HIV prevention interventions for AGYW in SSA, to ultimately enhance population impact and long-term sustainability.

- Findings from this systematic review highlight gaps in reporting of implementation outcome measures that could inform decisions around the translation and scale-up of EE HIV interventions targeting AGYW in SSA.

(Continued from previous page)

Conclusions: Results of the review emphasize the need for future economic empowerment HIV prevention interventions for AGYW in SSA to report multiple implementation strategies and highlight considerations for translating such programs into real-world settings. Researchers should pay close attention to reporting setting-level adoption, implementation cost, and intervention maintenance. These measures are needed for policy decisions related to the full merit and worth of EE HIV interventions and their long-term sustainability for AGYW.

Keywords: RE-AIM, Economic empowerment, Adolescent girls, Young women, Sub-Saharan Africa

\section{Background}

Across many countries in sub-Saharan Africa (SSA), adolescent girls live in a context of vulnerability and are exposed to a combination of intersecting systemic barriers based on their age, gender, education, ethnicity, socioeconomic status, and place of residence [1-3]. Every day, an estimated 1000 adolescent girls and young women aged 15-24 years are newly infected with human immunodeficiency virus (HIV) [4]. Globally, there are now 19.1 million adolescent girls and women living with HIV, of which $80 \%$ reside in sub-Saharan Africa [4]. Data from the Joint United Nations Program on HIV and AIDS (UNAIDS) estimates that three out of four new HIV infections in SSA among 15-19 years olds are among young women, and 7 out of 10 young women do not have comprehensive knowledge about HIV [5]. Additionally, the interactive effects of youth poverty and disease are particularly severe in SSA [6]. Decades of economic crisis across SSA have left millions of youth that are currently out of school unemployed [7-9]. These youth, particularly young girls, who miss out on education are more likely to engage in risk-taking behavior such as unprotected sex, transactional sex, and agedisparate sex [10-12]. Simultaneously, the population of adolescent girls and young women in SSA is expected to double from 100 million in 1990 to 200 million by 2020 [13]. This suggests a potential for new infections, and consequently a need to address the growing education and employment gap already faced by this population [14]. While there may be some challenges posed by the growing youth population in SSA, there are opportunities to optimize the demographic dividends from the "youth bulge", where more than half of the population is younger than 20 years, to foster youth employment and economic empowerment [15]. The potential for elevated infection rates among young females in SSA demonstrates an urgent need for sustainable programs that leverage on the capabilities of young people to avert new HIV infections in adolescent girls in high HIV risk settings [16]. If not properly addressed, the mutually reinforcing crisis of poverty and disease may threaten fragile development gains. The result of which is a devastating downward spiral in human development over the next generation for millions of adolescent girls and young women in the region.

Recognizing the urgency of the crisis, considerable research has been devoted over the past two decades to developing effective strategies to prevent HIV among adolescents and young people globally [17-20]. A number of theory-based prevention approaches targeting 
individual-level, group, community, and structural barriers to HIV have been implemented, with some targeting girls in schools [21-28] or within their communities [29-31] and some showing evidence of efficacy or effectiveness. HIV prevention interventions also led to the development of effective approaches to combat a spectrum of other health and behavioral problems, including depression, risky sexual behaviors, pregnancy intentions, and intimate partner violence [17, 32-35]. Examples of economic empowerment interventions include microfinance, vocational skills training, business development training, microenterprise development, cash transfers, and savings-led asset-based programs that work to alleviate girls' household economic hardships through the infusion of financial assets and resources [36-38]. Available evidence suggests that when implemented in conjunction with financial literacy curricula, such economic empowerment programs increase school attendance and personal savings among girls [39, 40]. Additionally, when these programs are combined with other social empowerment programs such as safe spaces, peersupport, and mentoring on female-specific issues related to health and well-being, they can increase girls' bargaining power, decrease their financial dependence on others, and reduce engagement in sexual risk-taking behaviors [41]. Combination HIV prevention interventions that include economic empowerment activities are particularly beneficial in low-resource settings such as SSA, where adolescent girls and young women are at increased risk to engage in transactional and crossgenerational sex due to limited economic assets [4244]. Numerous studies continue to show that women who lack economic independence are less able to negotiate safe sex with partners, less able to leave an abusive relationship, and are more likely to engage in transactional sex as means of survival $[45,46]$. These in turn increases their risk for HIV. Such evidence shows a strong link between economic instability and risky sexual behaviors that increase HIV risk among adolescent girls and young women in the region $[43,47]$.

Nevertheless, despite the increase in the number of these interventions targeting adolescent girls and young women in the region, it can take up to 17 years for these interventions to make their way to other adolescent girls underrepresented in scientific trials or in settings where its delivery could reasonably produce benefit [48, 49]. Additionally, a sizable gap remains between what is known about what works and how to effectively translate these interventions into practice [50,51]. One potential solution is the use of implementation science, and by this, we mean the scientific inquiry into what, why, and how interventions work in "real world" settings and to test approaches to improve them [52-54]. As described by Peters and colleagues, "implementation research seeks to understand and work within real-world conditions, rather than trying to control for these conditions" [55]. It also implies working with populations that will be affected by an intervention (i.e., adolescent girls themselves serving as an advisory board), rather than selecting beneficiaries who may not represent the target population of an intervention (such as studying only inschool girls or excluding girls who have comorbidities) $[54,56,57]$. One goal of implementation science is to appropriately expand the use of interventions that have been found efficacious and as broadly as feasible in order to foster the greatest public health impact $[51,58]$. The reach, effectiveness, adoption, implementation, and maintenance (RE-AIM) model is an implementation science framework for expanding interventions that have been found to be effective in research settings [58, 59]. The model focuses on the reach of the intervention to a representative proportion of the target population, the effectiveness of a program on specific outcomes, adoption of the program in a specified setting, and details of program implementation and maintenance $[58,59]$. To date, there are no published studies using the RE-AIM framework to evaluate the public health impact of economic empowerment HIV prevention programs for girls, and none of the published RE-AIM studies have looked at adolescent girls and young women populations in SSA.

The present study seeks to bridge this gap between research and practice in SSA. Our objectives are twofold: (1) to review the extent to which EE HIV prevention interventions for AGYW in SSA report on implementation outcomes, as conceptualized in the RE-AIM (reach, efficacy/ effectiveness, adoption, implementation, and maintenance) framework; and (2) to make recommendations for using the RE-AIM framework to advance the implementation of these interventions for girls and young women in the region. Through highlighting the reach, effectiveness, adoption, implementation, and maintenance of economic empowerment HIV interventions for adolescent girls and young women in SSA, we aim to assist researchers, practitioners, and policymakers in scaling up and evaluating new and existing economic empowerment interventions aimed at reducing the rate of new HIV infections.

\section{Methods}

A multi-step process was used to identify, review, and analyze existing economic empowerment HIV prevention interventions targeting adolescent girls and young women in SSA using the RE-AIM framework. For the purposes of this review, economic empowerment intervention was defined as a set of economic-related actions (i.e., microfinance, cash transfers, financial literacy, savings, and asset-based programs) [60, 61] with a coherent objective to bring about change or produce identifiable HIV prevention outcomes in three broad sectors: 


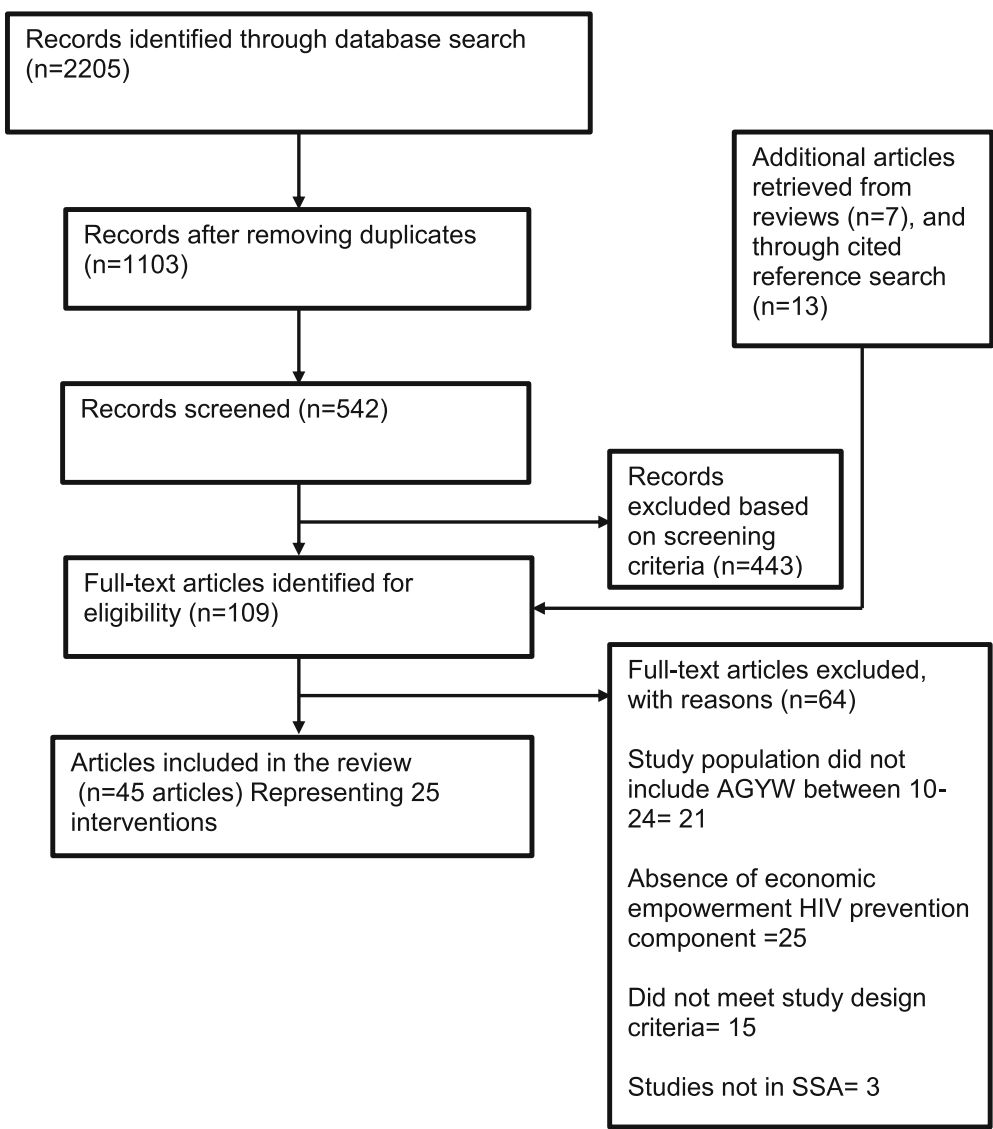

Fig. 1 Flow diagram of the search strategy. A total of 25 unique interventions reported in 45 articles were included in the review

health (i.e., girls' overall sexual and reproductive health, HIV and sexually transmitted infections (STIs), sexual risk-taking behaviors, pregnancy, and gender-related violence), social (i.e., education-related outcomes such as school attendance, employment, mental health, future outlook, etc.), and economic (savings, asset accumulation, small business, etc.).

\section{Search strategy}

A systematic search of the literature was executed from October 2018 to July 2019 to locate studies published in academic journals. Figure 1 outlines the search strategy, which was reported according to Preferred Reporting Items for Systematic Reviews and Meta-Analyses (PRISMA) guidelines (see Additional file 1). Two reviewers (JI and UN) independently searched PubMed, Ovid/MEDLINE, Science Direct, Ebscohost, PsycINFO, Scopus, and Web of Science databases with the following approximate search terms: (girls or young women) AND (HIV or AIDS) AND (prevention or intervention or program) AND (economic empowerment or microfinance or cash transfers or savings-led programs or asset-based programs) AND (sub-Saharan Africa or country-specific terms for each SSA country). The search teams were modified for each database. A detailed search strategy for the PubMed database is provided in Additional file 2. In addition, published systematic reviews focused on economic strengthening for HIV prevention, as well as reference lists from the included studies, were searched to augment the database literature search. The titles and abstracts of potentially relevant articles were independently screened by two reviewers (JI and UN) for eligibility. The full texts of articles that met the eligibility criteria were obtained and assessed by the two reviewers (JI and UN) independently for inclusion in the review. Discrepancies in the screening process and study eligibility were discussed and addressed based on consensus between the two reviewers (JI and $\mathrm{UN}$ ).

\section{Eligibility criteria}

Inclusion and exclusion criteria were developed to identify original research that empirically evaluated or tested economic empowerment strategies to prevent HIV among adolescent girls and young women in SSA. Articles were eligible for inclusion if they were (a) conducted in sub-Saharan Africa, (b) described an economic empowerment intervention with outcomes related to HIV prevention, (c) targeted adolescent girls and young women aged 10-24 or interventions that were not 
specific to AGYW but reported separately on AGYW, (d) written in English, and (e) published between 2000 and 2019. We included studies that used intervention designs ranging from randomized control trials to quasiand non-experimental evaluations of the interventions. Non-empirical studies (e.g., reviews, commentaries, editorials, and dissertations) and studies that did not explicitly assess the effect of economic empowerment on HIV prevention were excluded from the review.

\section{Data extraction}

For studies meeting the inclusion criteria, we extracted the following data: (1) title, author, country, study objective, and design; (2) information on the intervention being evaluated, including type of economic empowerment HIV prevention intervention, and target AGYW populations; (3) components of the intervention; and (4) RE-AIM framework implementation outcomes that included (a) reach (absolute number, proportion, and representativeness of AGYW in the economic empowerment HIV prevention interventions); (b) efficacy/effectiveness (impact of the intervention on AGYW HIV prevention behaviors, including overall sexual health factors, social factors, and economic outcomes); (c) adoption (absolute number, proportion, and settings participating in the intervention, and the extent to which the settings selected are representative of settings that the target population use or visit); (d) implementation (consistency of delivery as intended, time, and cost of implementation); and (e) maintenance (extent to which a program has become part of routine practice at the organizational level or the long-term effects of a program on outcomes at the individual level) [59].

\section{Data analysis}

Data from the articles included in this review were analyzed using narrative synthesis [62], with details on the reporting of the RE-AIM components synthesized. The articles included in this review are heterogeneous in terms of study design and measured outcomes; therefore, it was not practical to conduct a meta-analysis. To evaluate the included interventions within each dimension of the REAIM framework, two authors coded and scored each article independently using an adapted RE-AIM data extraction form that included a series of yes or no questions used to identify components within each of the RE-AIM dimension outcomes [63-65]. The adapted RE-AIM data extraction form is presented in Additional file 3. The form was used for calculating percentages of interventions meeting the criteria for the five RE-AIM dimensions (reach, efficacy/effectiveness, adoption, implementation, and maintenance). We summarized RE-AIM components using frequencies, proportions, and means. First, the frequencies and proportion of reported 26 components for each RE-AIM dimension were calculated separately for each study included in the review. Secondly, the average proportion of components within each RE-AIM dimension across the 25 unique interventions included in the review was calculated. The percentage and number of interventions reporting each RE-AIM dimension were reported to provide a comparable summary score across interventions.

\section{Risk of bias}

To systematically compare the interventions, we evaluated the rigor of each intervention using the Cochrane Collaboration risk-of-bias tool $[66,67]$. The tool consists of six domains: selection bias, performance bias, detection bias, attrition bias, reporting bias, and other bias $[66,67]$. The risk of bias was independently rated as low, high, or unclear by two authors using the guideline for each domain. The raters discussed each domain of the assessment tool to apply consistent judgment. If ratings differed, the rationale for the rating was discussed, and the study was re-reviewed to reach consensus. The Cochrane Collaboration risk of bias assessment tool was only used to evaluate the internal validity of the interventions included in the review; no study was excluded from the review based on the risk-of-bias score.

\section{Results \\ Study selection}

The initial database search yielded 2205 potentially relevant citations based on publication titles and abstracts (Fig. 1). A total of 542 papers were retrieved for full-text review for eligibility, and 443 were excluded. The most common reasons for exclusions were the absence of an economic empowerment HIV prevention intervention targeting adolescent girls and young women aged 1024 $(n=46)$, study location outside of sub-Saharan Africa $(n=3)$, or not meeting study design criteria $(n=15)$.

\section{Characteristics of included studies}

Forty-five articles were retained in the final group of articles. Most of the articles were pulled from the electronic data searches, except for 13 articles that were identified from the manual search of reference lists. The characteristics of the final included articles are presented in Table 1. We reported on 25 unique interventions that were highlighted across the forty-five articles included in the review. All 25 interventions reported in the articles were published between 2006 and 2018, thirteen interventions were conducted in Southern Africa, nine in East Africa, and one in Central Africa, West Africa, and North Africa respectively. The majority of AGYW targeted were between the ages of 15 and 24 years. Seventeen (68\%) interventions were randomized controlled trials, three (12\%) were cross-sectional interventions, three $(12 \%)$ were quasiexperimental designs, one (4\%) observational study, and 


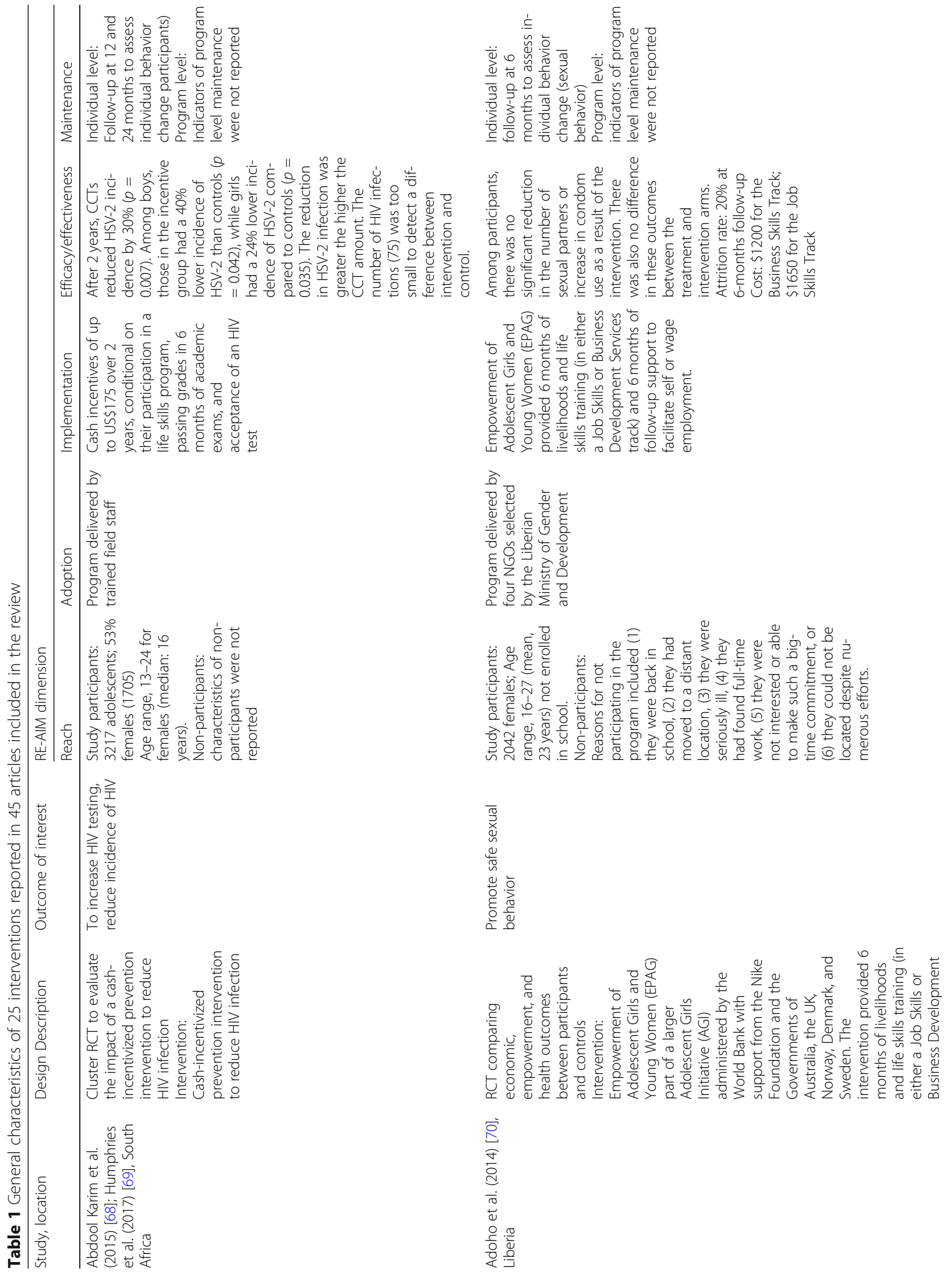




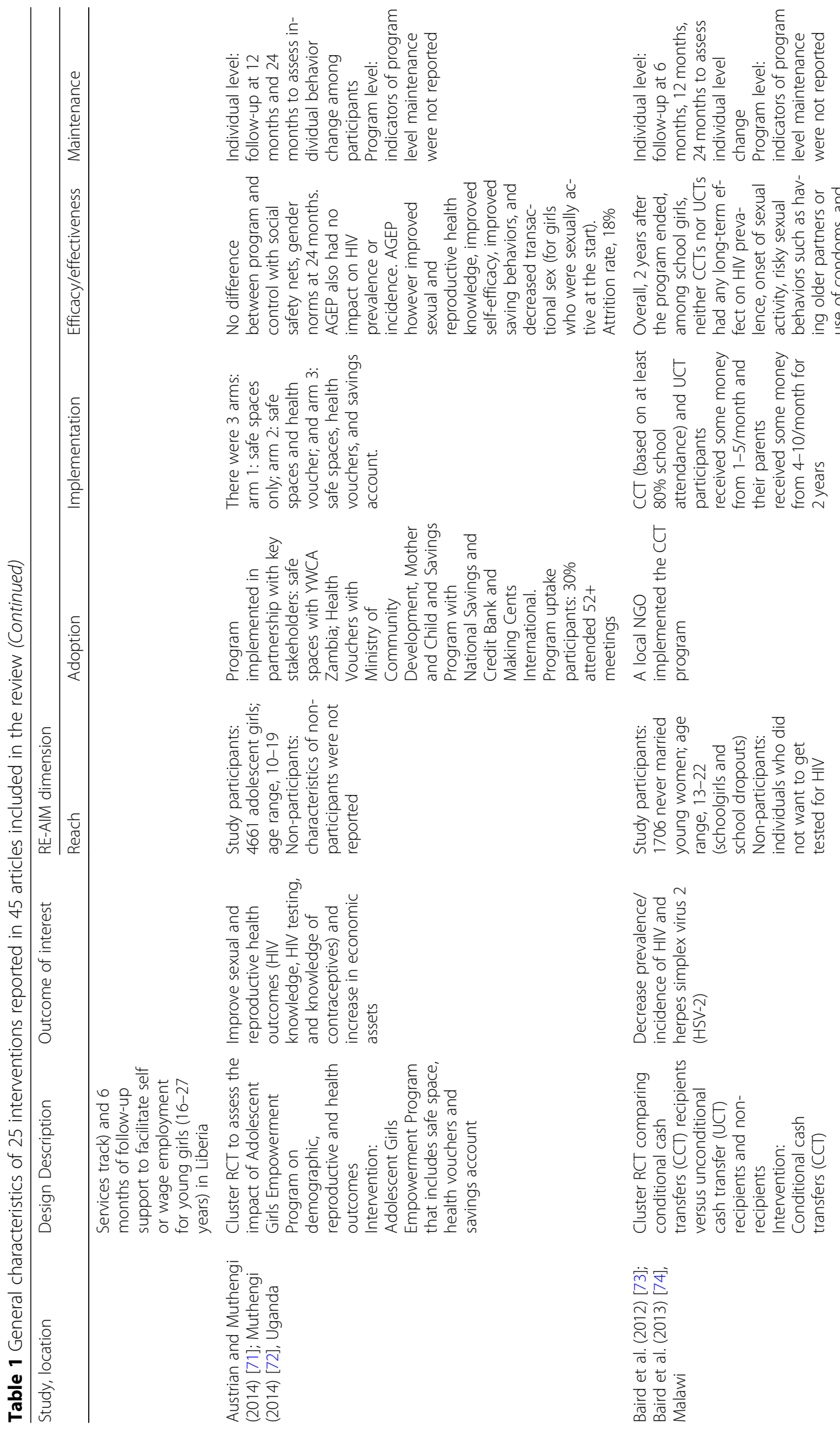




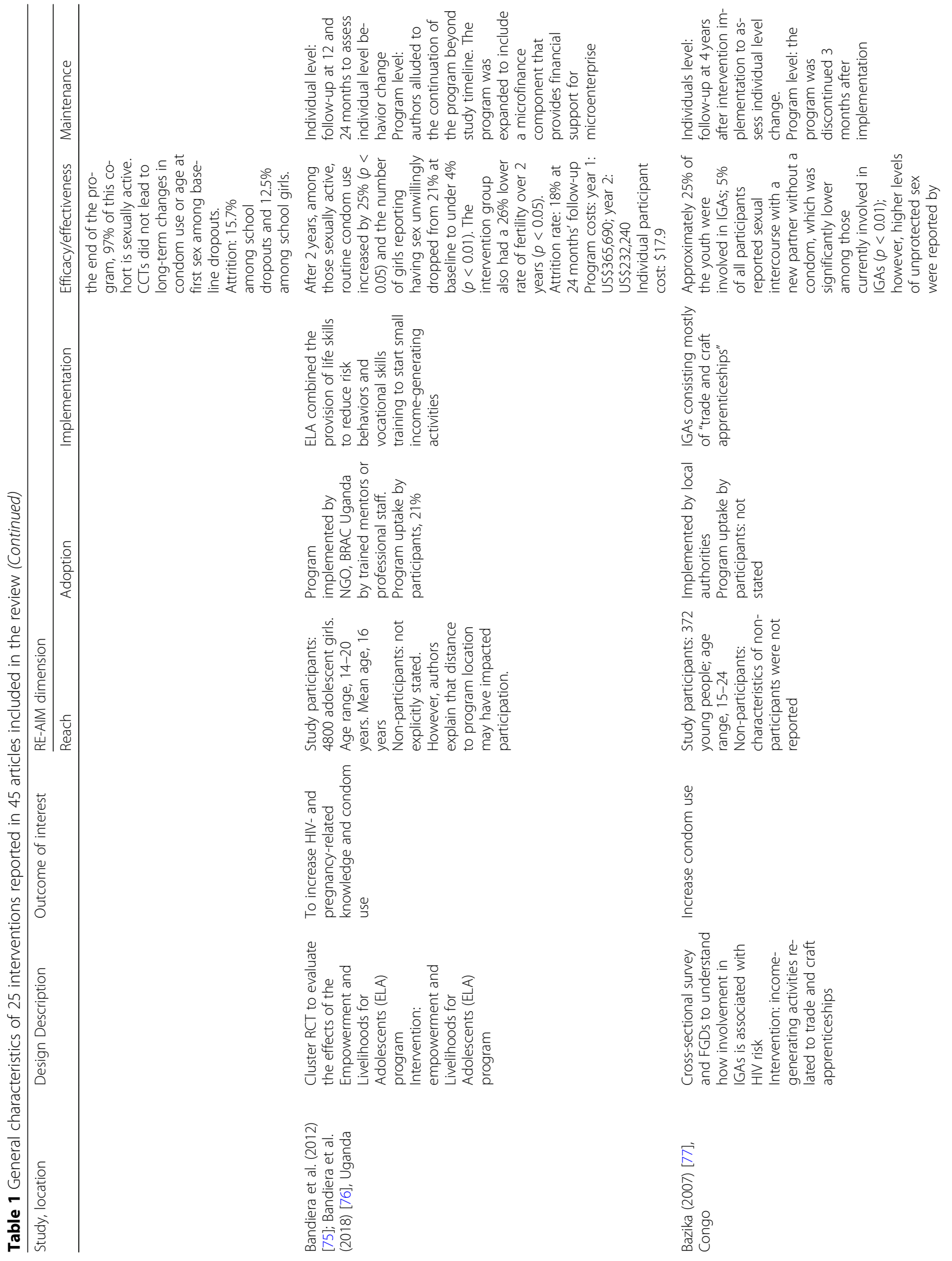




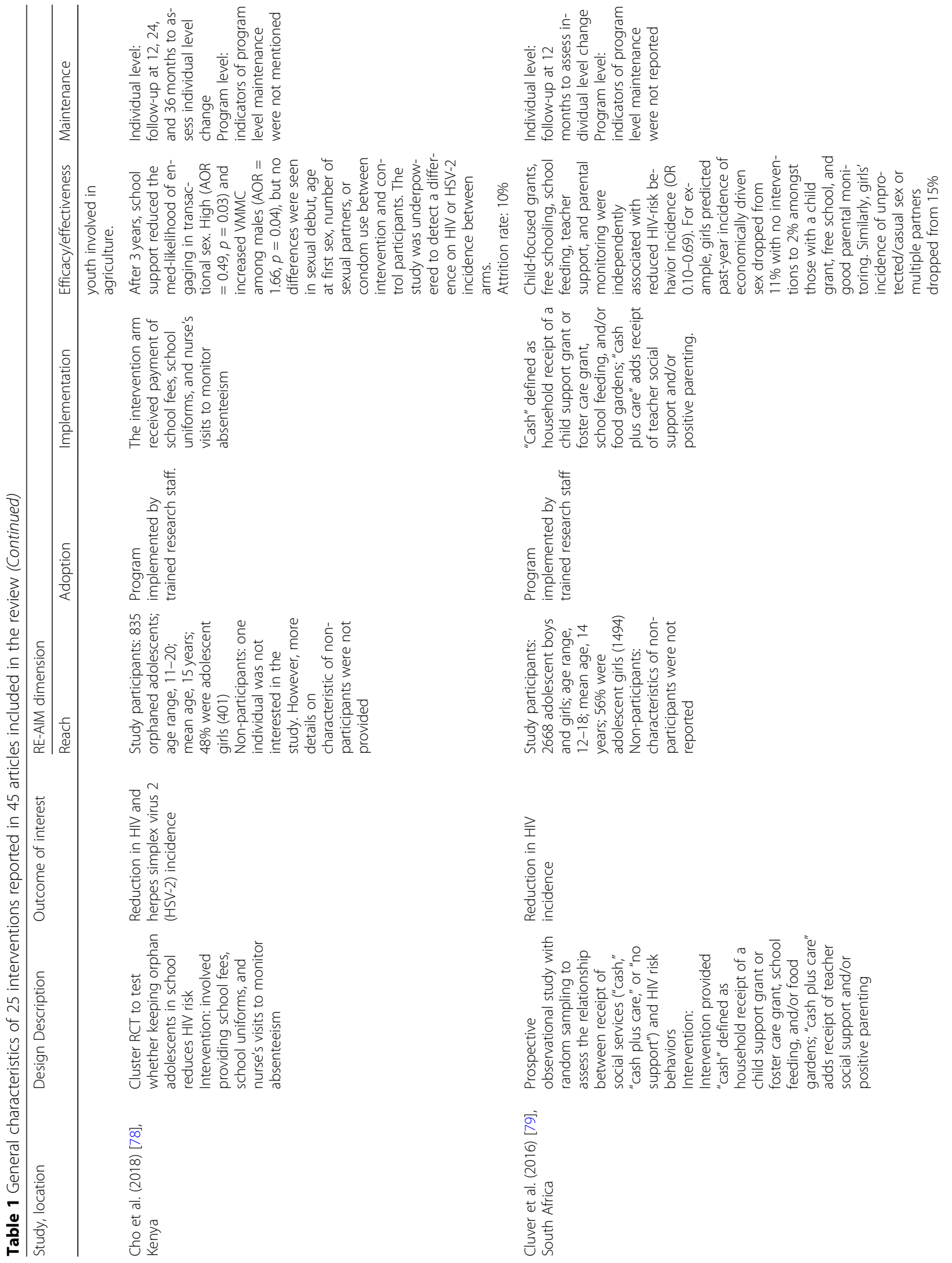




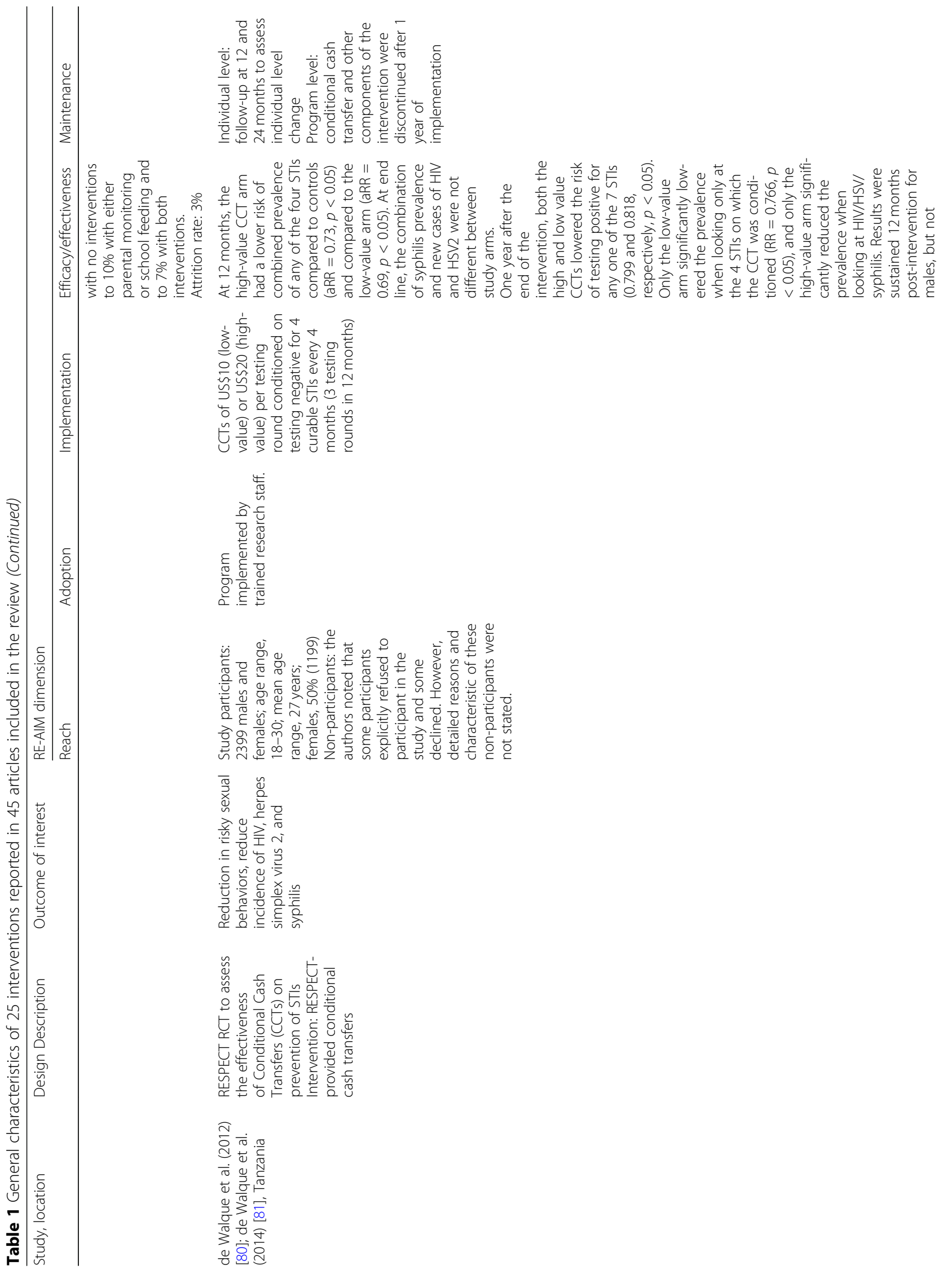




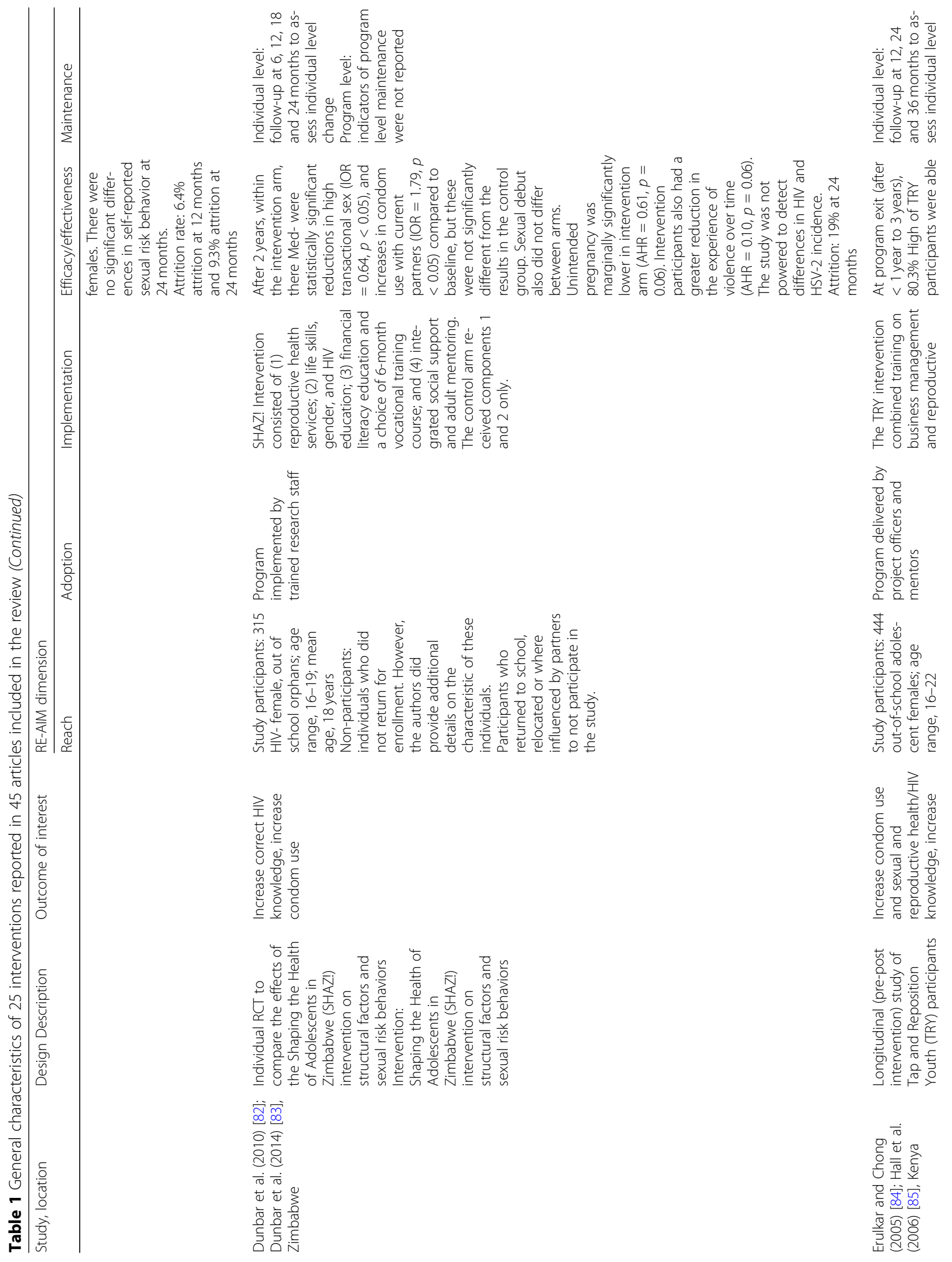




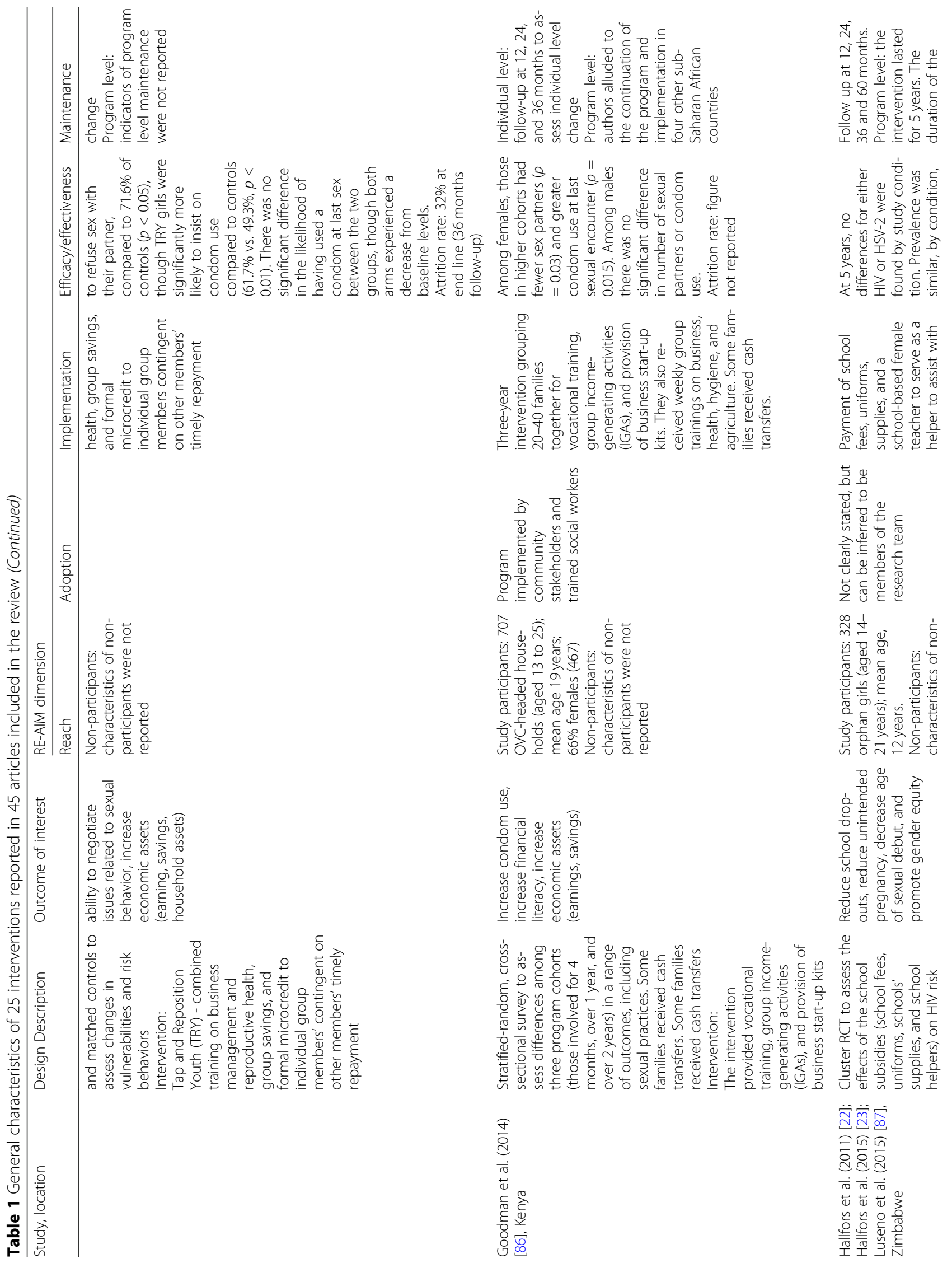




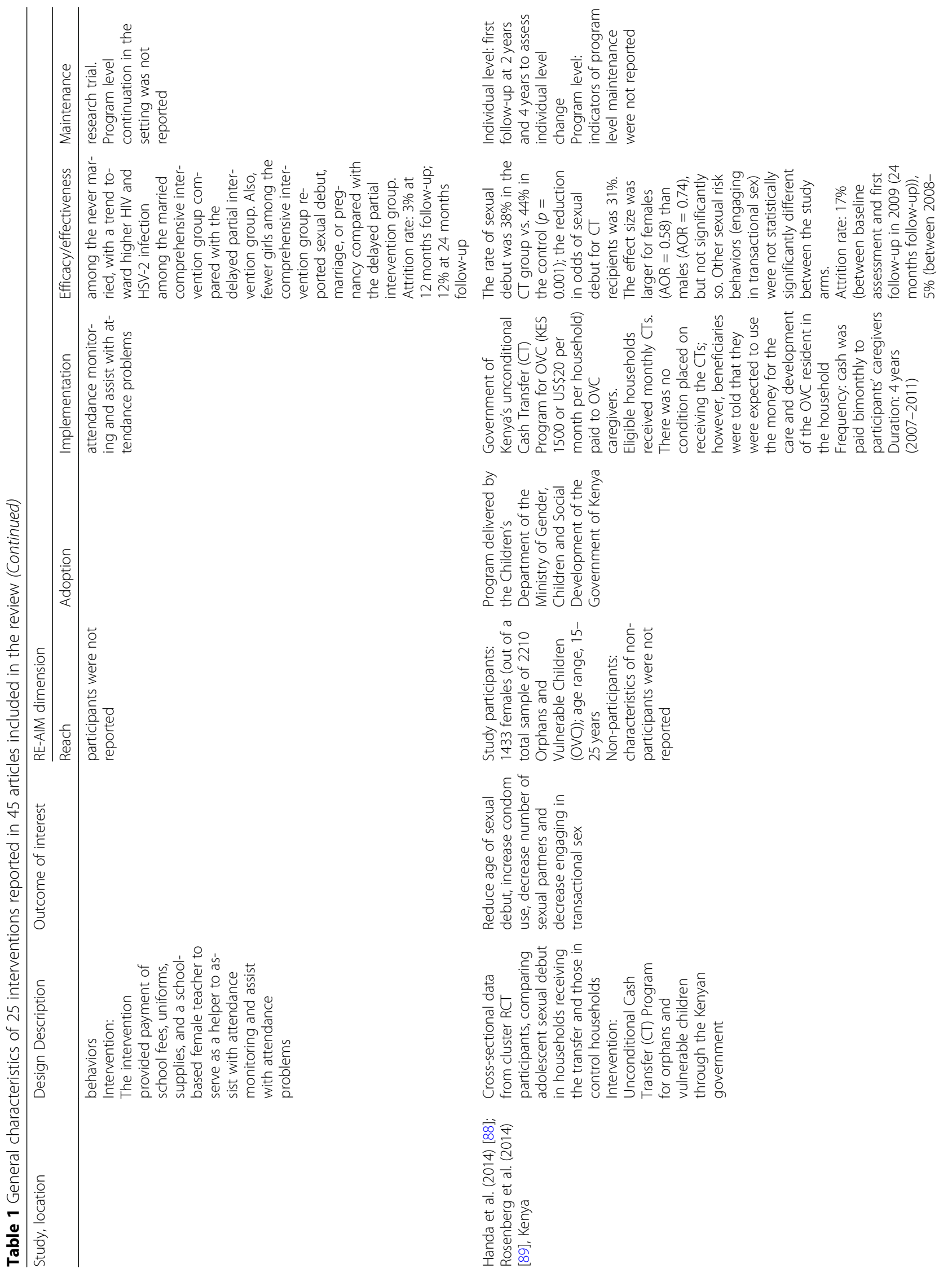




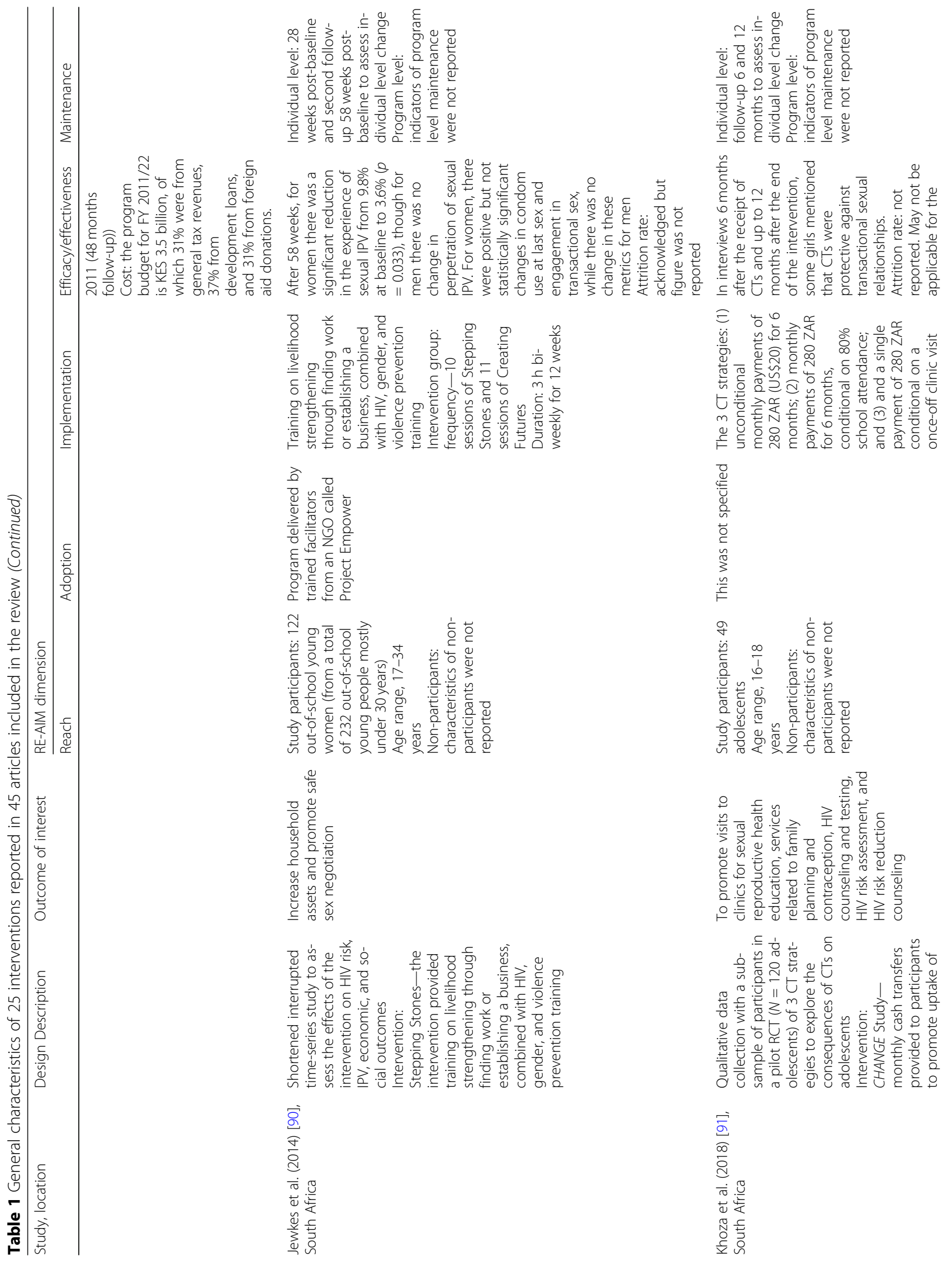




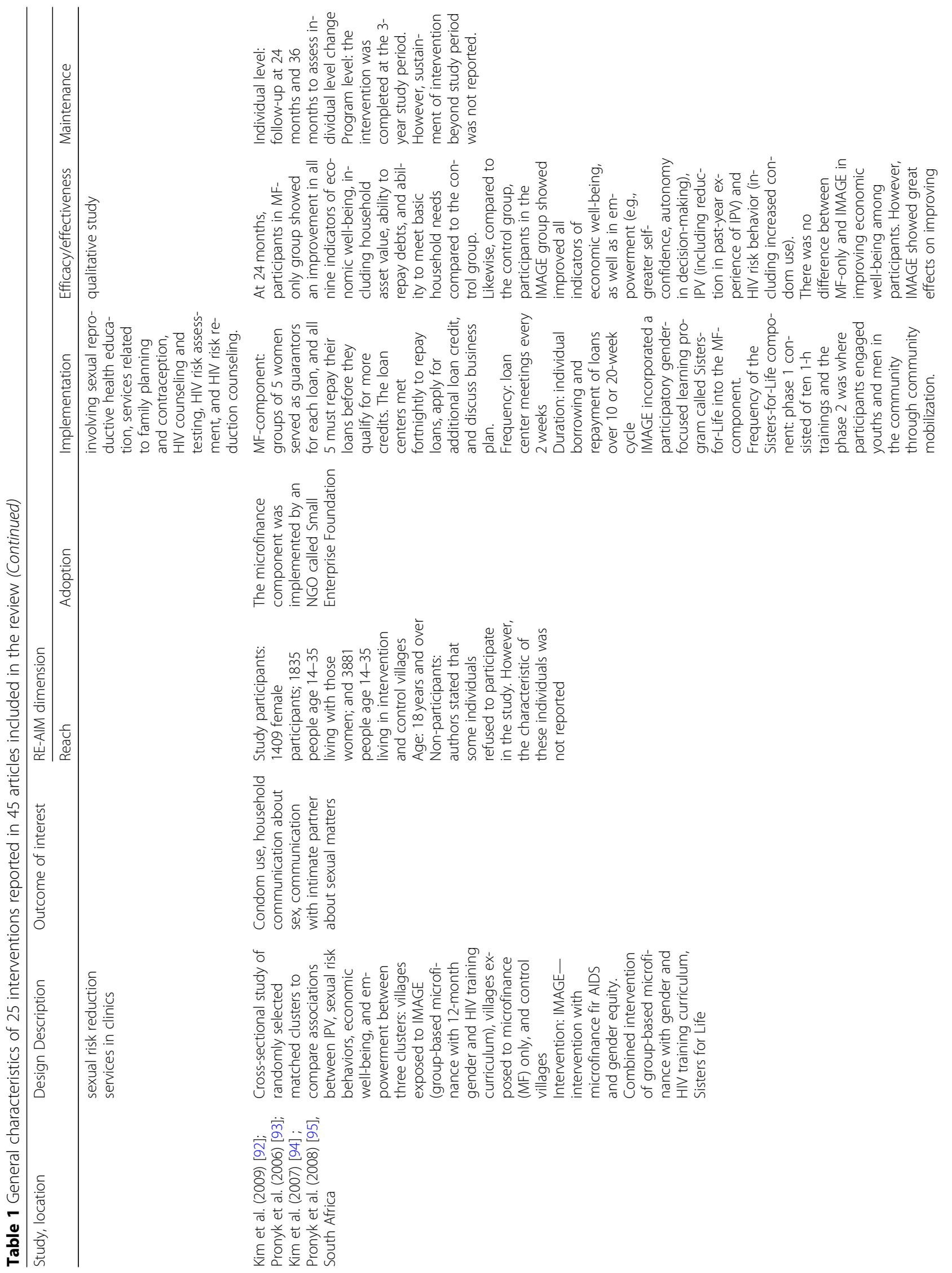




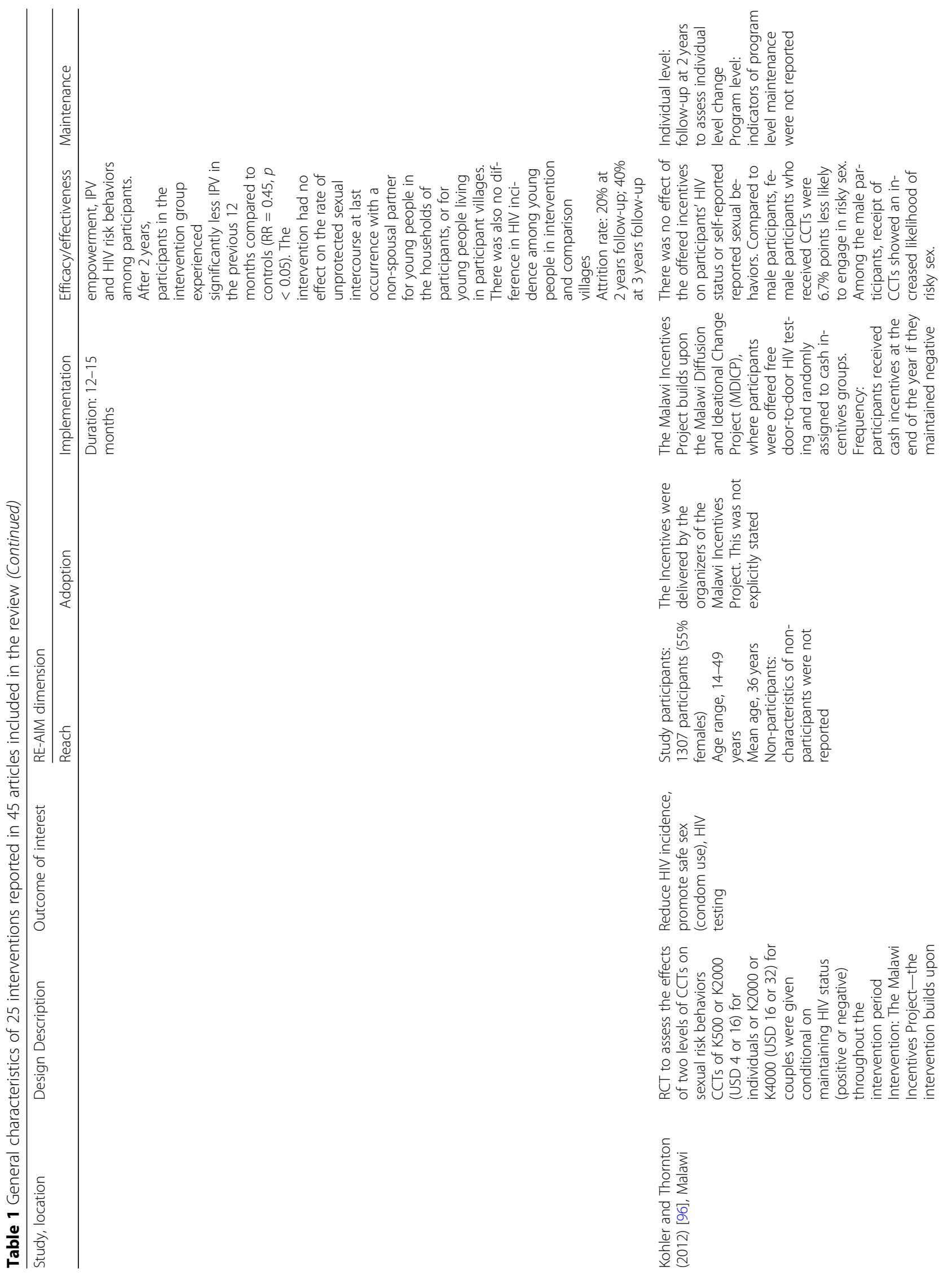




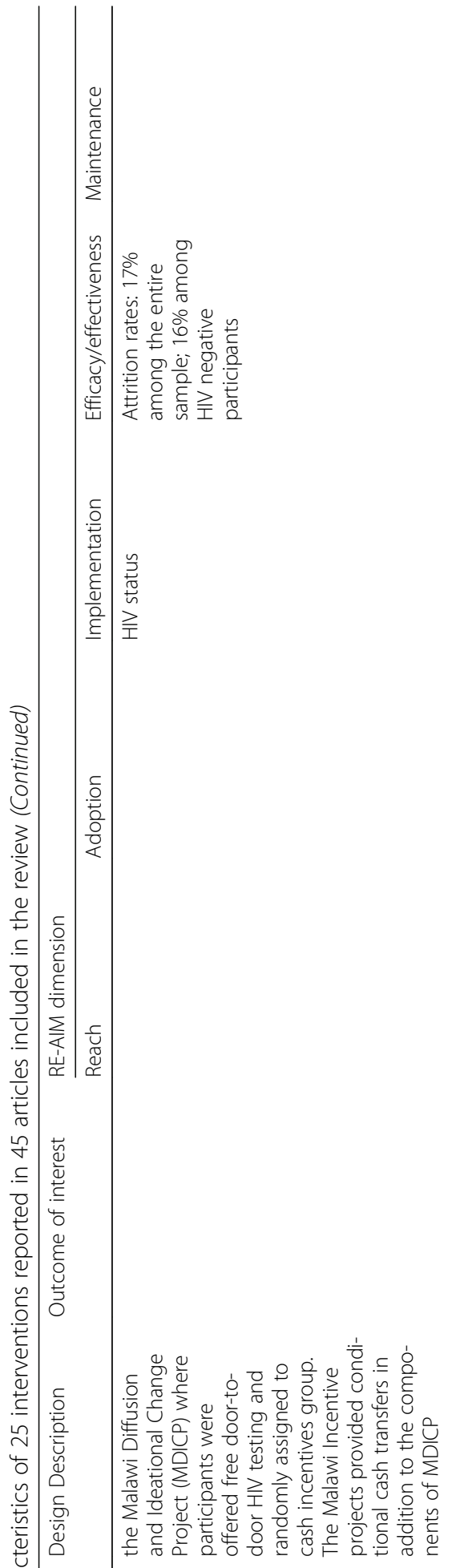

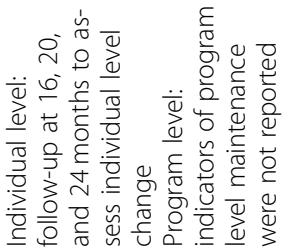

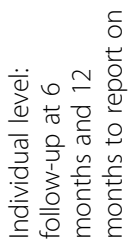

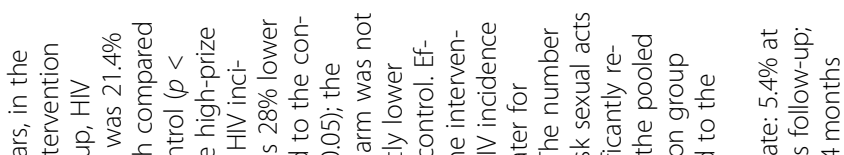

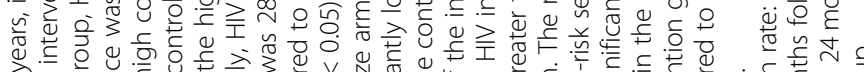
入ेo

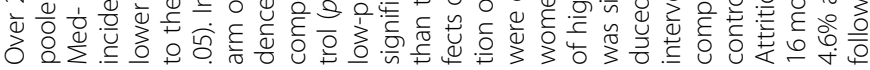

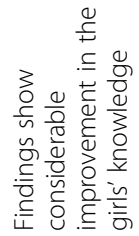

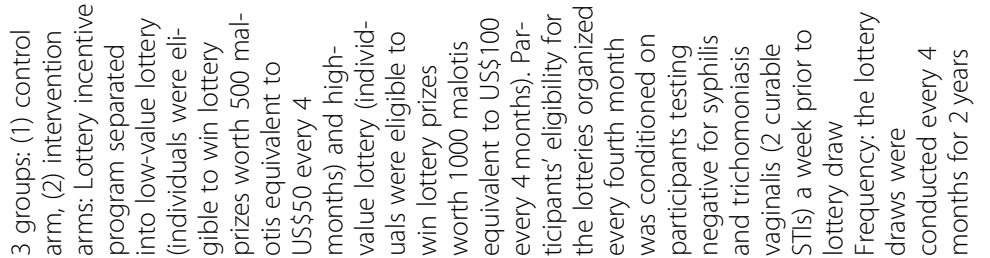

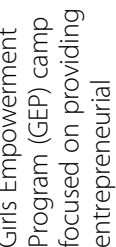

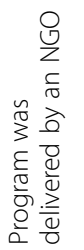

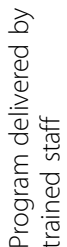

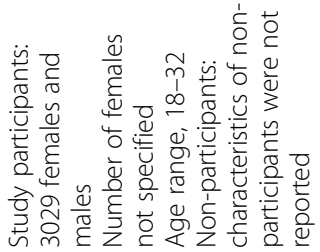

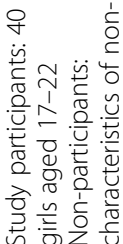

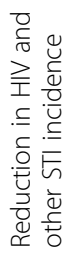

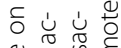

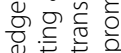

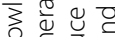

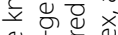

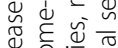

红.

윤

『

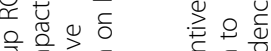

o.

i)

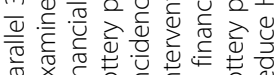

㐫玹语

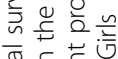

区.

足 氠

要

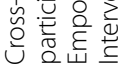

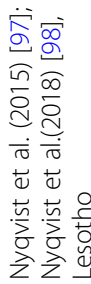

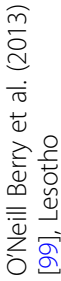




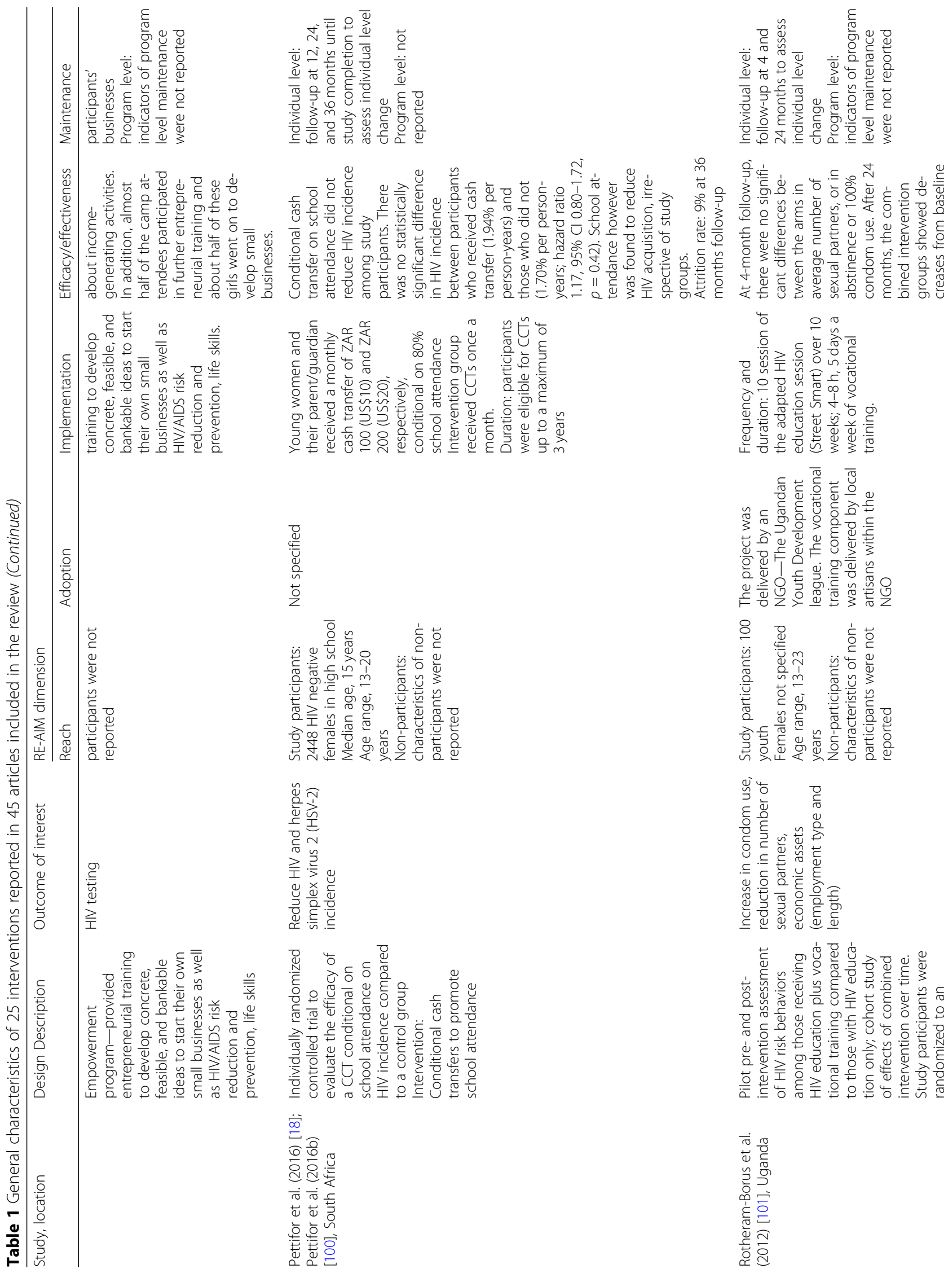



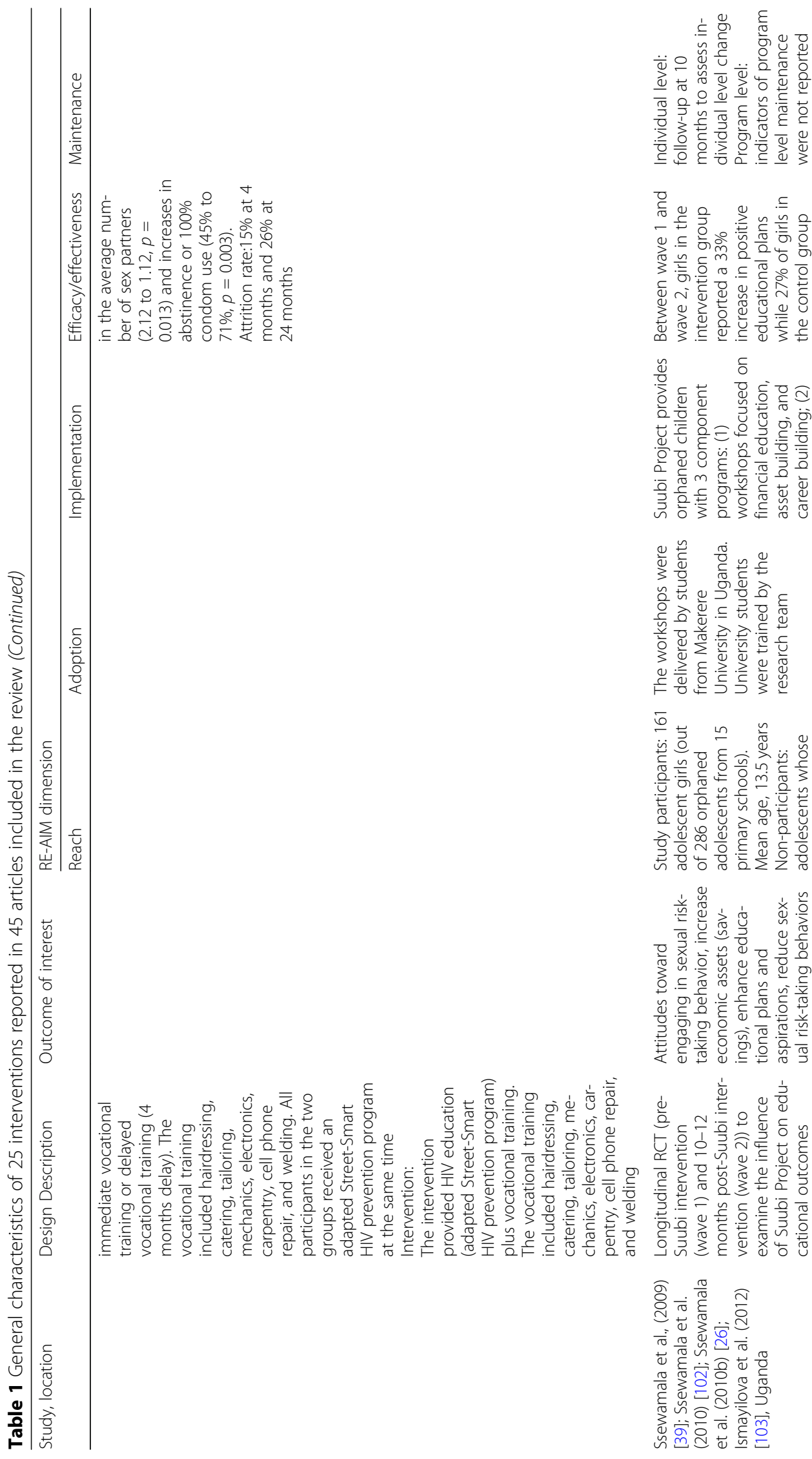

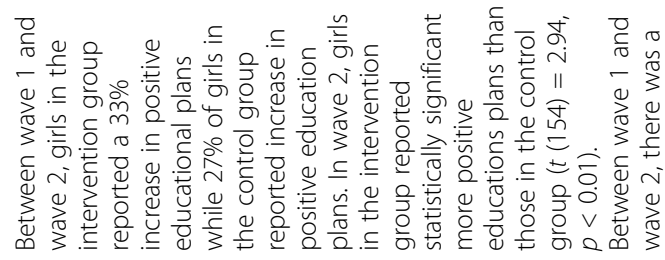

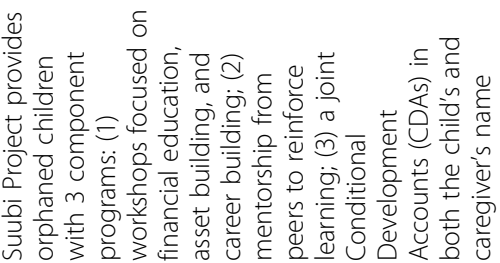

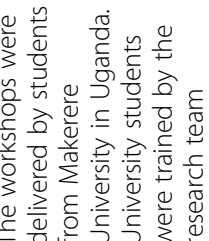
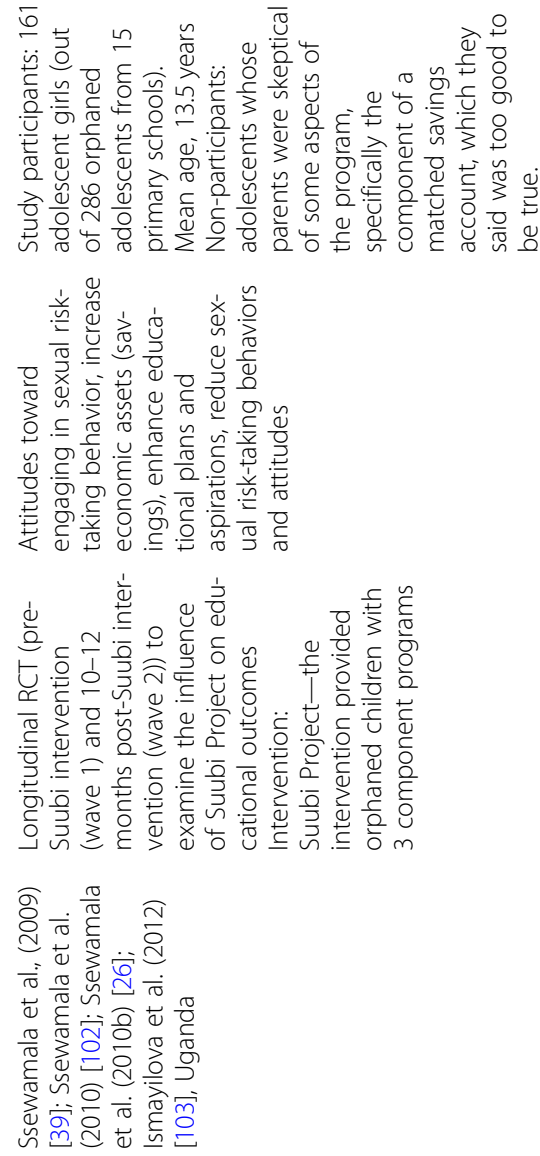


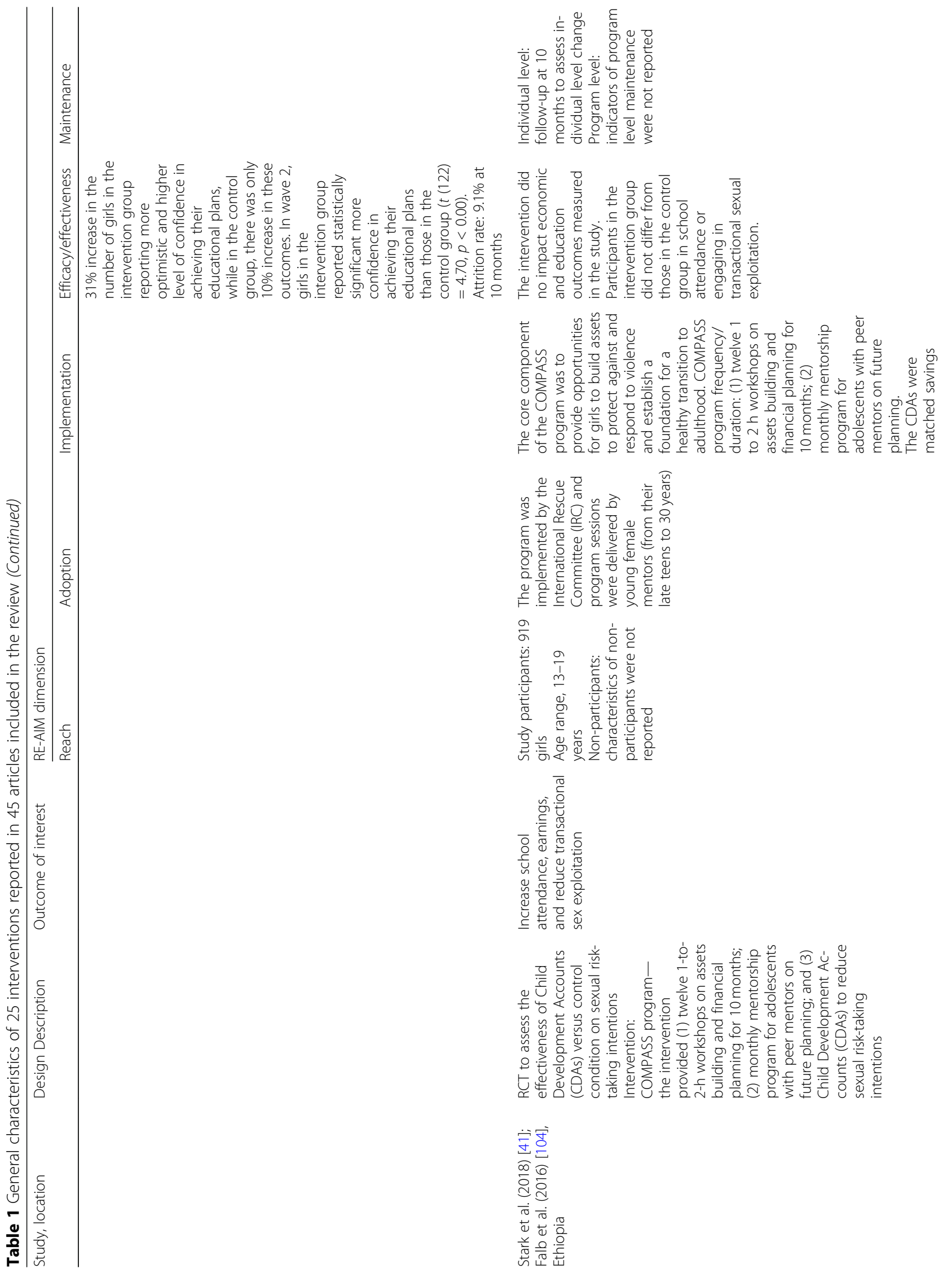




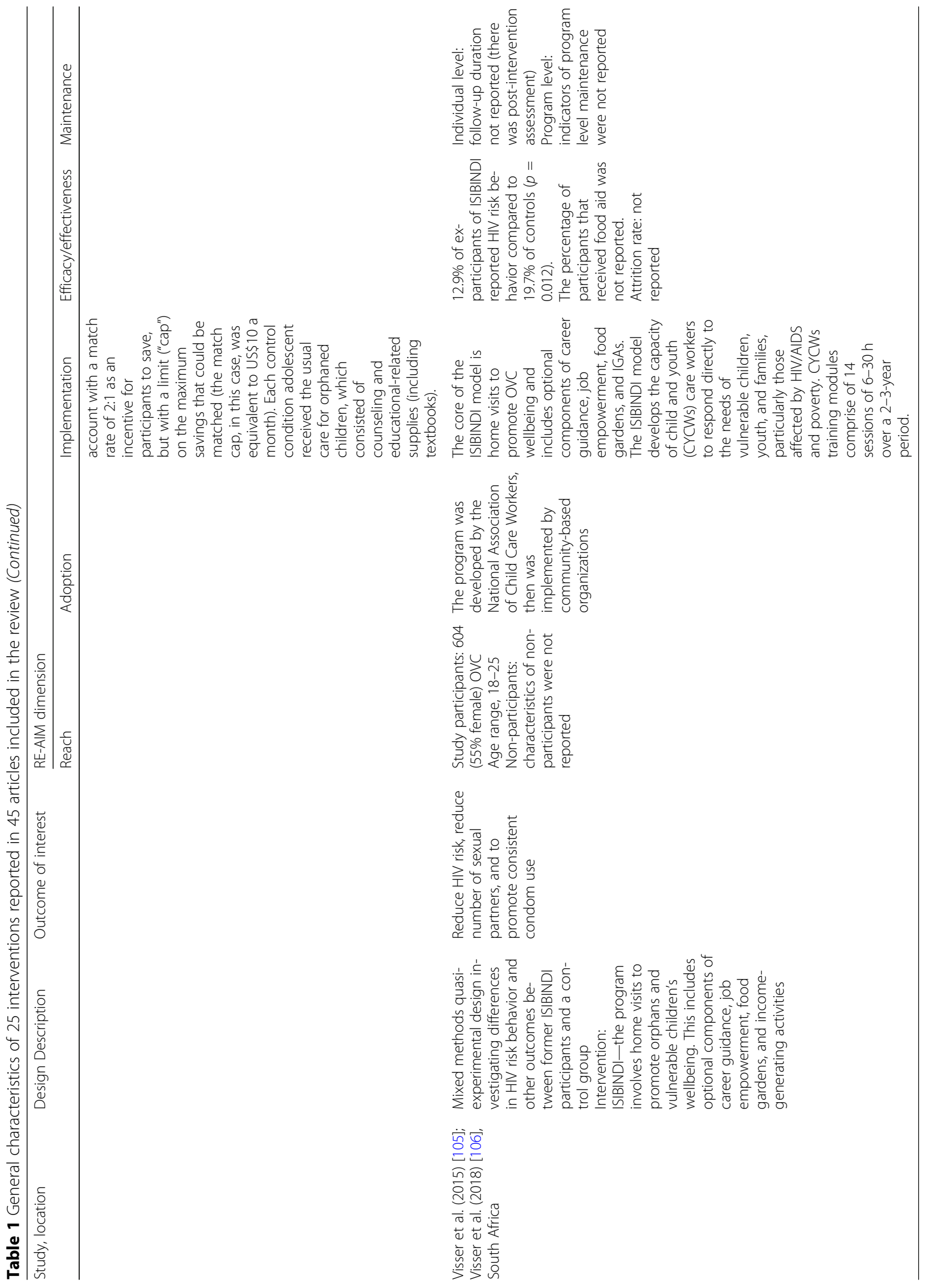


Table 2 Reporting on quality of included interventions (25 interventions reported in 45 papers included in the review)

\begin{tabular}{|c|c|c|c|c|c|c|c|c|c|}
\hline & $\begin{array}{l}\text { Selection } \\
\text { bias } \\
\text { (random } \\
\text { sequence } \\
\text { generation) }\end{array}$ & $\begin{array}{l}\text { Selection } \\
\text { bias } \\
\text { (allocation } \\
\text { concealment) }\end{array}$ & $\begin{array}{l}\text { Performance } \\
\text { bias }\end{array}$ & $\begin{array}{l}\text { Detection } \\
\text { bias }\end{array}$ & $\begin{array}{l}\text { Attrition } \\
\text { bias } \\
\text { (incomplete } \\
\text { outcome } \\
\text { data) }\end{array}$ & $\begin{array}{l}\text { Reporting } \\
\text { bias } \\
\text { (selective } \\
\text { reporting) }\end{array}$ & $\begin{array}{l}\text { Other } \\
\text { sources } \\
\text { of bias }\end{array}$ & $\begin{array}{l}\% \text { risk } \\
\text { of } \\
\text { bias }\end{array}$ & Comments \\
\hline $\begin{array}{l}\text { Abdool Karim et al. } \\
\text { (2015) [68]; } \\
\text { Humphries et al., } \\
\text { (2017) [69], South } \\
\text { Africa }\end{array}$ & Low risk & Low risk & Low risk & Low risk & Unclear & Low risk & Low risk & $14.3 \%$ & $\begin{array}{l}\text { Study design: } \\
\text { quantitative (comparison } \\
\text { of treatment and control } \\
\text { groups) }\end{array}$ \\
\hline $\begin{array}{l}\text { Adoho et al. (2014) } \\
\text { [70], Liberia }\end{array}$ & Low risk & Unclear & Low risk & Low risk & Low risk & Low risk & Unclear & $28.6 \%$ & $\begin{array}{l}\text { Study design: } \\
\text { quantitative (comparison } \\
\text { of two treatment groups } \\
\text { to a control group) }\end{array}$ \\
\hline $\begin{array}{l}\text { Austrian and } \\
\text { Muthengi (2014) [71]; } \\
\text { Muthengi (2014) [72], } \\
\text { Uganda }\end{array}$ & High risk & Unclear & Low risk & Low risk & Low risk & Low risk & Unclear & $42.9 \%$ & $\begin{array}{l}\text { Study design: mixed } \\
\text { methods (comparison of } \\
\text { two treatment groups to } \\
\text { a control group) }\end{array}$ \\
\hline $\begin{array}{l}\text { Baird et al. (2012) } \\
\text { [73]; Baird et al. } \\
\text { (2013) [74], Malawi }\end{array}$ & Low risk & Low risk & Low risk & Low risk & Unclear & Low risk & Low risk & $14.3 \%$ & $\begin{array}{l}\text { Study design: mixed } \\
\text { methods (pre- and post- } \\
\text { test comparison for inter- } \\
\text { vention and control } \\
\text { groups) }\end{array}$ \\
\hline $\begin{array}{l}\text { Bandiera et al. (2012) } \\
\text { [75]; Bandiera et al. } \\
\text { (2018) [76], Uganda }\end{array}$ & Low risk & Unclear & Low risk & Low risk & Low risk & Low risk & Low risk & $14.3 \%$ & $\begin{array}{l}\text { Study design: } \\
\text { quantitative (pre- and } \\
\text { post-test comparison for } \\
\text { intervention and control } \\
\text { groups) }\end{array}$ \\
\hline $\begin{array}{l}\text { Bazika (2007) [77], } \\
\text { Congo }\end{array}$ & Unclear & Unclear & Low risk & Low risk & Unclear & Unclear & Unclear & $71.4 \%$ & $\begin{array}{l}\text { Study design: } \\
\text { quantitative (pre- and } \\
\text { post-test assessment of } \\
\text { intervention participants) }\end{array}$ \\
\hline $\begin{array}{l}\text { Cho et al. (2018) [78], } \\
\text { Kenya }\end{array}$ & Low risk & Unclear & Low risk & Low risk & Low risk & Low risk & Low risk & $14.3 \%$ & $\begin{array}{l}\text { Study design: } \\
\text { quantitative (comparison } \\
\text { between intervention } \\
\text { and control groups) } \\
\text { Longitudinal study with } \\
\text { annual repeated } \\
\text { measures over } 4 \text { years }\end{array}$ \\
\hline $\begin{array}{l}\text { Cluver et al. (2016) } \\
\text { [79], South Africa }\end{array}$ & Low risk & Unclear & Low risk & Low risk & Low risk & Low risk & Low risk & $14.3 \%$ & $\begin{array}{l}\text { Study design: } \\
\text { quantitative (comparison } \\
\text { between cash alone and } \\
\text { integrated cash plus care } \\
\text { intervention for HIV-risk } \\
\text { reduction) } \\
\text { Prospective longitudinal } \\
\text { study }\end{array}$ \\
\hline $\begin{array}{l}\text { de Walque et al. } \\
\text { (2012) [80]; de } \\
\text { Walque et al. (2014) } \\
\text { [81], Tanzania }\end{array}$ & Low risk & High risk & Low risk & Low risk & Low risk & Low risk & Low risk & $14.3 \%$ & $\begin{array}{l}\text { Study design: } \\
\text { quantitative (pre- and } \\
\text { post-test comparison for } \\
\text { intervention and control } \\
\text { groups) }\end{array}$ \\
\hline $\begin{array}{l}\text { Dunbar et al. (2010) } \\
\text { [82]; Dunbar et al. } \\
\text { (2014) [83], } \\
\text { Zimbabwe }\end{array}$ & Low risk & High risk & Low risk & Low risk & Low risk & Low risk & Low risk & $14.3 \%$ & $\begin{array}{l}\text { Study design: } \\
\text { quantitative (pre- and } \\
\text { post-test comparison for } \\
\text { intervention and control } \\
\text { groups) }\end{array}$ \\
\hline $\begin{array}{l}\text { Erulkar and Chong } \\
\text { (2005) [84]; Hall et al. } \\
\text { (2006) [85], Kenya }\end{array}$ & High risk & High risk & Low risk & Low risk & Low risk & Low risk & Unclear & $42.9 \%$ & $\begin{array}{l}\text { Study design: } \\
\text { quantitative (pre- and } \\
\text { post-assessment of inter- } \\
\text { vention participants) } \\
\text { Longitudinal study }\end{array}$ \\
\hline Goodman et al. & Low risk & Unclear & Low risk & Low risk & Unclear & Low risk & Low risk & $28.6 \%$ & Study design: \\
\hline
\end{tabular}


Table 2 Reporting on quality of included interventions (25 interventions reported in 45 papers included in the review) (Continued)

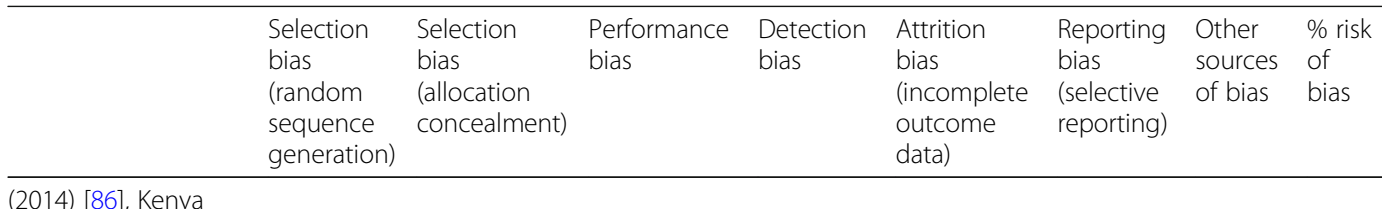

(2014) [86], Kenya

Hallfors et al. (2011)

[22]; Hallfors et al.

(2015) [23]; Luseno

et al. (2015) [87],

Zimbabwe

Handa et al. (2014)

[88]; Rosenberg et al.

(2014) [89], Kenya

Low risk

Unclear

Low risk

Low risk

Low risk

(1)

Jewkes et al. (2014)

[90],South Africa

High risk

High risk

Low risk

Low risk

Unclear

Low risk

(1)

Khoza et al. (2018)
[91], South Africa

Kim et al. (2009) [92]: Low risk

Pronyk et al. (2006)

[93]; Kim et al. (2007)

[94]; Pronyk et al.

(2008) [95], South

Africa

Kohler and Thornton Low risk (2012) [96], Malawi

Nyqvist et al. (2015) Low risk

[97]; Nyqvist

et al.(2018) [98],

Lesotho

O'Neill Berry et al.,

(2013) [99], Lesotho

Unclear

High risk

High risk

Low risk

Low risk

Low risk

Low risk

Low

Low risk

sis

Low risk High risk

(n)


Table 2 Reporting on quality of included interventions (25 interventions reported in 45 papers included in the review) (Continued)

\begin{tabular}{|c|c|c|c|c|c|c|c|c|c|}
\hline & $\begin{array}{l}\text { Selection } \\
\text { bias } \\
\text { (random } \\
\text { sequence } \\
\text { generation) }\end{array}$ & $\begin{array}{l}\text { Selection } \\
\text { bias } \\
\text { (allocation } \\
\text { concealment) }\end{array}$ & $\begin{array}{l}\text { Performance } \\
\text { bias }\end{array}$ & $\begin{array}{l}\text { Detection } \\
\text { bias }\end{array}$ & $\begin{array}{l}\text { Attrition } \\
\text { bias } \\
\text { (incomplete } \\
\text { outcome } \\
\text { data) }\end{array}$ & $\begin{array}{l}\text { Reporting } \\
\text { bias } \\
\text { (selective } \\
\text { reporting) }\end{array}$ & $\begin{array}{l}\text { Other } \\
\text { sources } \\
\text { of bias }\end{array}$ & $\begin{array}{l}\% \text { risk } \\
\text { of } \\
\text { bias }\end{array}$ & Comments \\
\hline & & & & & & & & & intervention group \\
\hline $\begin{array}{l}\text { Ssewamala et al., } \\
\text { (2009) [39]; } \\
\text { Ssewamala et al. } \\
\text { (2010) [102]; } \\
\text { Ssewamala et al. } \\
\text { (2010b) [26]; } \\
\text { Ismayilova et al. } \\
\text { (2012), Uganda }\end{array}$ & Low risk & Unclear & Low risk & Low risk & Low risk & Low risk & Low risk & $14.3 \%$ & $\begin{array}{l}\text { Study design: } \\
\text { quantitative (pre- and } \\
\text { post-comparison of inter- } \\
\text { vention and control } \\
\text { groups) }\end{array}$ \\
\hline $\begin{array}{l}\text { Stark et al. (2018) } \\
\text { [41]; Falb et al. (2016) } \\
\text { [104], Ethiopia }\end{array}$ & Low risk & Unclear & Low risk & Unclear & High risk & Low risk & Unclear & $57.1 \%$ & $\begin{array}{l}\text { Study design: } \\
\text { quantitative (pre- and } \\
\text { posttest comparison for } \\
\text { intervention and control } \\
\text { groups). } \\
\text { Reported null findings } \\
\text { that the intervention did } \\
\text { not seem to keep the } \\
\text { participants in school, nor } \\
\text { influence out-of-school } \\
\text { girls to return to school }\end{array}$ \\
\hline $\begin{array}{l}\text { Visser et al. (2015) } \\
\text { [105]; Visser et al. } \\
\text { (2018) [106], South } \\
\text { Africa }\end{array}$ & High risk & Unclear & Low risk & High risk & High risk & Low risk & Unclear & $71.4 \%$ & $\begin{array}{l}\text { Study design: mixed } \\
\text { method (quasi- } \\
\text { experimental post- } \\
\text { intervention assessment } \\
\text { between intervention } \\
\text { and control group and } \\
\text { focus group discussions). } \\
\text { Utilized focus group dis- } \\
\text { cussions to generate in- } \\
\text { formation on strategies } \\
\text { to sustain ISIBINDI } \\
\text { intervention }\end{array}$ \\
\hline
\end{tabular}

one (4\%) time-series design study. Six types of economic empowerment (EE) interventions were in the included in the review: cash transfers (conditional or unconditional) $[18,41,68,73,80,86,88,91,96,97,107]$, job skills or business development $[70,84,86,99,108]$, matched savings account $[71,102]$, vocational skills training [75, 77, 82, 101, 105], payment of school fees and school supplies [23, 78], and loan credit [92]. Several HIV prevention outcomes were targeted, including reduction in HIV incidence [18, $22,68,80,82,97]$, increase in condom use [70, 73, 75, 77, $78,83,84,86,90,92,96,101,107]$, reduction in the number of sexual partners $[70,73,78,86,101,107]$, reduction in transactional sex $[41,71,78,82,88,90,91]$, increase in sexual and reproductive health knowledge [71], selfefficacy $[71,84]$, and delay in sexual debut $[22,73,78,88$, $101]$.

\section{Quality of evidence}

The quality assessment of the selected articles is reported in Table 2. The level of bias varied widely, with a range of $0.0 \%$ to $71.4 \%$ risk among the interventions.
Among the interventions using quantitative methods, one of the interventions [18] was found to have a $0.0 \%$ (low) risk of bias. The risk of bias for quantitative methods ranged from $0.0 \%$ (low) [18] to $71.4 \%$ (high) [77]. Among interventions using mixed methods, one of the interventions [92] was also found to have a $0.0 \%$ (low) risk of bias. The risk of bias for mixed methods interventions also ranged from $0.0 \%$ (low) [92] to $71.4 \%$ (high) [99]. The only qualitative study in the review had a high risk of bias (71.4\%) [91].

The most common strengths of the interventions that utilized quantitative methods were: the ability to conduct a longitudinal follow-up of study participants over time, the random selection and assignment of participants, and the reporting of descriptive intervention details. However, one of the common weaknesses was the limited use of intent-to-treat analysis, although attrition was acknowledged by the majority of the interventions [18, 22, 41, 70, $71,75,78-80,83,84,88,92,96,97,101,102]$. For the interventions that utilized mixed-methods [71, 73, 90, 92, $99,105]$, common strengths were the ability to triangulate 
Table 3 Proportion of interventions reporting RE-AIM dimensions and components

\begin{tabular}{|c|c|c|}
\hline RE-AIM dimensions and components & Reporting frequency $(n=25)$ & Reporting proportion (\%) \\
\hline \multicolumn{3}{|l|}{ Reach } \\
\hline Method to identify target population & 24 & 96.0 \\
\hline Inclusion criteria & 16 & 64.0 \\
\hline Exclusion criteria & 3 & 12.0 \\
\hline Sample size & 25 & 100.0 \\
\hline Participation rate & 9 & 36.0 \\
\hline Characteristics of participants & 25 & 100.0 \\
\hline Characteristics of non-participants & 8 & 32.0 \\
\hline Representativeness & 18 & 72.0 \\
\hline${ }^{a}$ Average of overall reach dimensions & 16.00 & 64.00 \\
\hline \multicolumn{3}{|l|}{ Efficacy/effectiveness } \\
\hline Measures/results for at least one follow-up & 24 & 96.0 \\
\hline Intent to treat utilized & 5 & 20.0 \\
\hline Quality-of-life measure & 23 & 92.0 \\
\hline Baseline activity measured & 25 & 100.0 \\
\hline Percent attrition & 16 & 64.0 \\
\hline 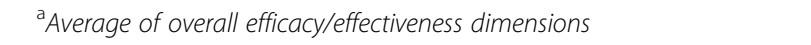 & 18.60 & 74.4 \\
\hline \multicolumn{3}{|l|}{ Adoption } \\
\hline Description of intervention location & 24 & 96.0 \\
\hline Description of staff who delivered intervention & 22 & 88.0 \\
\hline Method to identify target delivery agent & 16 & 64.0 \\
\hline Level of expertise of delivery agent & 19 & 76.0 \\
\hline Adoption rate & 3 & 12.0 \\
\hline${ }^{a}$ Average of overall adoption dimensions & 16.80 & 67.2 \\
\hline \multicolumn{3}{|l|}{ Implementation } \\
\hline Intervention duration and frequency & 25 & 100.0 \\
\hline Extent protocol delivered as intended & 0 & 0.0 \\
\hline Measures of cost of implementation & 3 & 12.0 \\
\hline${ }^{a}$ Average implementation dimensions & 9.33 & 37.3 \\
\hline \multicolumn{3}{|l|}{ Maintenance } \\
\hline \multicolumn{3}{|l|}{ Individual-level maintenance } \\
\hline Was individual behavior assessed $\geq 6$ months post-intervention & 23 & 92.0 \\
\hline Was individual behavior assessed $\geq 24$ months post-intervention ${ }^{a}$ & 3 & 12.0 \\
\hline Was individual behavior assessed $\geq 48$ months post-intervention ${ }^{a}$ & 4 & 16.0 \\
\hline \multicolumn{3}{|l|}{ Program-level maintenance } \\
\hline Indicators of program continuation & 2 & 8.0 \\
\hline Some measure/discussion of alignment with organization/setting & 1 & 4.0 \\
\hline${ }^{a}$ Average of overall maintenance dimensions & 6.60 & $26.4 \%$ \\
\hline
\end{tabular}

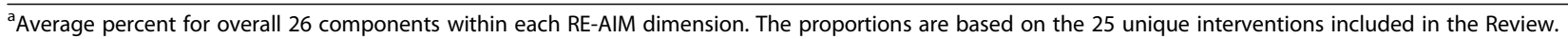
Components were included to ensure relevance with HIV prevention health behavior change

data obtained from qualitative and quantitative methods and providing additional explanation for the quantitative data using qualitative data. The strength of the qualitative studies were the use of detailed quotes and narratives to explain study findings.

\section{Reporting of RE-AIM dimensions}

The reporting of RE-AIM dimensions was assessed using a previously developed and validated data extraction tool that included implementation outcome components based on the RE-AIM framework $[59,63]$. Across all the 
interventions, average reporting rates (defined here as the overall percent of components) were highest for efficacy/effectiveness $\approx 19(74.4 \%)$ and adoption $\approx 17(67.2 \%)$, followed by reach $16(64.0 \%)$, and lowest for implementation $\approx 9(37.3 \%)$ and maintenance $\approx 7(26.4 \%)$. Table 3 provides details on each of the components assessed across the RE-AIM framework and a summary of the overall percentage of interventions reporting on each of the REAIM dimensions. The reporting status for the 26 components for the RE-AIM dimensions per study is provided in Additional file 2.

\section{Reach}

The average proportion reporting on the reach components was $16(64.0 \%)$. The sample size and participants' characteristics were the most frequently reported item $25(100 \%)$. All interventions reported on sample size, defined as the number of participants who consented to participate in the study/intervention. Of the interventions that recruited only AGYW $(n=12)[18,22,41,70$, $72,73,75,84,87,92,99]$, sample size ranged from $n=40$ in the O'Neill Berry and colleagues study [99] to $n=4800$ in the study by Bandiera and colleagues [75]. In interventions that recruited other populations in addition to AGYW, the sample size ranged from $n=46$ to $n=6576$ (with the AGYW sample size being between 122 and 1705). Five (20\%) interventions [77, 91, 96, 97, 101] did not explicitly report the sample size for AGYW, although it was mentioned that AGYW were included in the study. Participant characteristics included; reports on age, gender (for interventions that included other population), employment status, education attainment, and socioeconomic status (measured as household income in some interventions). The next commonly reported reach component was the method for identifying the target population for the study and this was assessed in $24(96.0 \%)$ of the studies reviewed [18, 22, 39, 41, 68, $70,71,73,75,77,78,80,82,84,86,88,90-92,96,97$, $99,101,105]$. Most of the interventions were conducted in Southern Africa, specifically in South Africa where 7 $[18,68,79,90-92,105]$ out of the $25(28 \%)$ interventions were located. The description of methods utilized to identify the target population varied across interventions from single-sentence descriptors to detailed reporting of the protocol used. Strategies utilized to identify the target population included using schools, youth centers, and community stakeholders. Regarding factors that foster or hinder the ability to reach the target audience, stakeholders' engagement and school recruitment were emphasized as beneficial strategies to enhance reach.

Sixty-four percent(16) of the interventions [18, 26, 41, $70,73,75,80,83,84,86,88,90-92,96,97]$ reported study participants' inclusion criteria. Only 3(12.0\%) studies $[18,73,80]$ explicitly stated participants' exclusion criteria. Participant inclusion criteria were typically related to participants' age, place of residence, membership (e.g., being part of the school), parental status (being an orphan), and gender. Individuals were mainly excluded if they did not meet the inclusion criteria for the interventions. Participation rate was reported in nine(36.0\%) [18, $68,70,75,79,83,90,92,96]$ of the included interventions. The participation rate ranged from $21 \%$ to $97.50 \%$. Eighteen $(72.0 \%)$ of the interventions $[18,41,68,70,73$, $75,78,80,83,84,86,88,90-92,97,102,107]$ reported on the representativeness of recruited study participants' relative to the target population. This was determined based on comparing demographic characteristics (e.g., age, education level) of study participants to those of the target population. Reporting on this component allowed the researchers to assess the extent to which the intervention could be generalizable across the target population and setting. The rigor of the study design was reported as an indicator of representativeness. Interventions that utilized randomized controlled trials reported representativeness as one of the strengths of their studies. According to the RE-AIM framework, studies should describe the characteristics of participants of the target population in comparison with non-participants. Eight(32\%) of the interventions provide some form of information on the characteristics of individuals who did not participate in their study. Some of the reasons for non-participation included unavailability of individuals (e.g., going back to school and having full-time jobs), inability to complete study procedures (e.g., not wanting to test for HIV, not returning for study procedure, and not obtaining consents from parents), limited access to the study location (e.g., distance from the individuals' residence to study site was a barrier to participating and geographic relocations) and lack of interest in the study.

\section{Efficacy/effectiveness}

Efficacy/effectiveness was the most consistently reported RE-AIM dimension across all interventions (74.4\%). Twenty four(96.0\%) interventions reported on at least one post-intervention effect; 5(20.0\%) interventions used intent-to-treat analyses and the remainder analyzing only data from participants who completed the intervention. All interventions included in the review included HIV prevention measures as primary outcomes. HIV prevention measures included; reduction in $\mathrm{HIV}$ incidence $[22,68,79,80]$, reduction in number of sexual partners [70, 73, 79, 86], condom use $[70,73,75,77,78,83,84,86,90,92,101]$, decrease in transactional sex $[41,71,78,83,89-91]$, and sexual debut $[23,73,78,83,87,88,97]$. Of the 25 interventions that measured HIV prevention outcomes, $20(80 \%)$ reported that the economic empowerment HIV prevention intervention resulted in 
statistically significant positive changes in HIV prevention outcomes.

Sixteen $(64.0 \%)$ interventions reported their percent attrition $[22,70,71,75,78-80,83,84,88,92,96,97,100-$ $102]$, which ranged from $5 \%$ to $40 \%$. Attrition rates were examined in relation to participants' loss to follow-up and non-use of the intervention [75]. Reasons for attrition included; participants' relocation, death, change of phone number, and logistics challenges. In terms of logistics challenges, Erulkar and Chong [84] reported some delays in participants receiving their loans and accessing their savings account as a result of limited human resources which accounted for some of the attritions they faced. Some participants were concerned that they may not have access to their savings account or loans; therefore, they dropped out of the study. In addition, Bandiera and colleagues [75] examined how participants' characteristics influence attrition between the intervention and control groups and found that married AGYW in the intervention were less likely to be tracked at follow-up. A high proportion of the interventions $23(92.0 \%)$ reported on participants' quality of life $[18,22,39,41,70,71,73,75,77,78$, $80,82,84,88,90-92,96,97,99,101,105]$ and found that economic empowerment HIV prevention interventions generally improved quality of participants lives and did not have any significant negative outcomes.

\section{Adoption}

The average proportion reporting on adoption components was $17(67.2 \%)$. Twenty-two of the interventions [22, 39, 41, $68,70,71,73,75,78,80,82,84,86,88,90,92,96,97,99$, $101,105]$ provided some description of the staff who delivered the intervention. Interventions were delivered by a range of staff with different levels of expertise and included research assistants, community leaders, and organization staff. Staff responsibilities included delivering parts of the interventions that consisted of moderating the discussion and intervention meeting groups, distributing conditional cash incentives, training participants' income-generating skills, and educating participants on intervention curriculum (e.g., sexual and reproductive health training, financial training, and income-generating skills and crafts). Seventysix percent (19) of the interventions explicitly stated implementing staff level of expertise [22, 39, 41, 68, 70, 71, 73, $75,80,82,84,86,88,90,92,99,101,105]$, but for those that were not stated, it could be inferred from their job titles or their organization's focus. Sixteen(64.0\%) interventions reported on the methods used to identify staff who delivered the intervention $[22,70,71,73,75,78,80,82,86,88,90$, $92,97,99,101,105]$. Intervention staff were mainly identified through their participation in the research project or collaborating organization.

The most commonly reported adoption component was the description of intervention location, reported by
24(96.0\%) studies $[18,22,39,41,68,70,71,73,75,78$, $80,82,84,86,88,90-92,96,97,99,101,105]$. Intervention locations included schools, community centers, and refugee camps. These locations were identified as typical settings that the target population visit or use. Also, most of the interventions were restricted to a specific geographical area. Most of the interventions were implemented in one site. The least reported adoption component was the adoption rate. Only $3(12.0 \%)$ interventions reported on intervention adoption rate among participants $[86,99,101]$. There were no reports on setting level adoption rates.

\section{Implementation}

The average proportion reporting on implementation components was about 9(37.3\%). All 25(100.0\%) interventions reported on the format of the intervention; specifically, they provided information on intervention duration and frequency $[18,22,39,41,68,70,71,73,75$, $77,78,80,82,84,86,88,90-92,96,97,99,101,105]$. Intervention ranged in duration from a single session to two or more (up to 14) sessions. None of the interventions explicitly reported on fidelity or the extent to which the intervention protocol was delivered as intended.

The cost of delivering the intervention was mentioned in only three (12.0\%) interventions [70, 73, 75]. Implementation cost items included skills training cost $[70,75]$, administrative cost $[73,75]$, and cost of monetary incentive $[73,75]$. Two interventions [70, 75] further conducted cost-benefit analyses to determine if the benefits/returns from the interventions for the participants outweighed the cost of implementing the interventions. These two interventions assessed intervention benefit based on the number of participants who participated in the incomegenerating component of the intervention. The authors highlighted that equipping AGYW with skills to generate sustainable income, which would in return reduce their chance of engaging in risky sexual behaviors [70, 75]. Specifically, Adoho and colleagues [70] found that the value provided by the program was equivalent to a 3 year increase in income among EE program participants. The study by Bandiera and colleagues [75] reported gains/benefits to the economic empowerment intervention in the form of delaying early marriage and childbirth and improving HIV and pregnancy-related knowledge.

\section{Maintenance}

The average proportion reporting on maintenance components was about $7(26.4 \%)$. Among the maintenance components, individual-level indicators were reported more frequently than program-level indicators. Twentythree (92.0\%) interventions reported at least one followup measure, particularly the primary outcomes at 6 
months $[18,22,39,41,68,70,71,73,75,77,78,80,82$, $84,86,88,91,92,96,97,99,101]$. The longest follow-up period reported was 24 months after baseline assessment [71]. The majority of the post-intervention assessments were conducted within 12 to 24 months after completion of the intervention. There were a few interventions that had follow-up assessments beyond 24 months after intervention completion; 6 for 36 months [18, 22, 78, 84, 86] follow-up, 2 for 48 months [88, 89] follow-up, and 1 for 60 months follow-up [23].

In terms of program-level maintenance, six interventions reported $[22,75,77,80,86,92]$ on indicators of program level maintenance or sustainability. Only two(8\%) interventions explicitly stated that the interventions were sustained beyond the study period $[75,86]$ For one study, the intervention was adapted to fit the context by including an additional component [75]. Two $(8 \%)$ interventions were discontinued before the study period end date [77, 80], and another two(8\%) ended at the completion of the study period [22, 92]. For the two interventions that were completed at the end of the study period, it was not clear if they were sustained beyond the study period.

\section{Discussion}

The primary aim of this review was to systematically assess the implementation of economic empowerment HIV prevention programs for AGYW in SSA. This review goes beyond an assessment of intervention effectiveness to report implementation outcomes as conceptualized by the RE-AIM framework. The RE-AIM framework was used as a guideline to determine the impact of EE HIV prevention interventions for AGYW. We evaluated five key components important for the translation of research findings to practice: reach, effectiveness, adoption, implementation, and maintenance [59, 109]. These components are important in understanding the factors that influence, not only adoption, but the cost and sustainability of economic empowerment interventions as a strategy for HIV prevention among AGYW in SSA.

A total of 25 (reported in 45 papers) economic empowerment interventions among AGYW were identified, described, and evaluated based on the five RE-AIM dimensions. On average, the included interventions reported on $14(53.86 \%)$ of the 26 components that constitute the RE-AIM dimensions. Major knowledge gaps exist relating to reporting of implementation and maintenance (least reported RE-AIM dimensions) of economic empowerment HIV interventions for AGYW in SSA. Specifically, the interventions in the review mainly focused on reporting intervention-specific components (e.g., sample size, intervention location, and effectiveness), with minimal reporting of broad or system- level components such as implementation costs, program-level sustainability, and intervention fidelity. Although concerning, the underreporting of broad or system-level elements is consistent with reports from other systematic reviews using the RE-AIM framework [110-115] that also found limited reporting of these dimensions. This further confirms the previous report on the predominant focus on intervention effectiveness, with limited attention to external factors that may impact the translation of effective interventions to realworld settings. Researchers need to also focus on reporting broad or system-level measures as well as intervention-specific measures. Broad or systemlevel factors are critical with understanding how findings from interventions apply to local settings, population, and available resources [116]. It informs the overall relevance and appropriateness of these interventions in realworld settings, and the potential for health gains by reducing HIV incidence among AGYW in SSA.

Reporting on intervention reach is important to inform future dissemination of interventions that have been found to be effective or efficacious towards behavior change. To scale-up economic empowerment HIV prevention intervention, there is the need to understand how to reach target populations. In this review, participants' characteristics and sample sizes were consistently reported across interventions. This is congruent with previous reviews on HIV prevention interventions $[32,117]$ that reported frequent reporting of participants characteristics such as their demographics. Some of the interventions in the review specified the degree to which the target samples were representative of the larger population. Information on the external population from which a study sample is drawn from helps to inform the generalizability of the findings to a larger population [111]. However, the characteristics of non-participants and participants, as well as the reasons for non-participation, were rarely reported in the interventions. This limits the understanding of contextual factors that may influence AGYW participation in such interventions. With scant information on characteristics of non-participants, researchers may be missing individuals who are most in need of these interventions, such as AGYW residing in remote areas, rural areas, and those with low literacy. To enhance the translation of intervention to a wider population, researchers should improve on the reporting of the characteristics of non-participants as this may extend program reach and inclusivity.

Consistent with past reviews, intervention effectiveness was the most commonly reported RE-AIM element across all interventions, with baseline activity measures reported for all included interventions [110, 111]. The outcome measures included; HIV incidence, number of 
sexual partners, condom use, transactional sex, and sexual debut. Findings from this systematic review highlight the impact on economic empowerment intervention on HIV reduction among AGYW, with about $19(74.7 \%)$ of the interventions reporting statistically significant improvements on HIV risk reduction measures among intervention participants compared with controls. For effectiveness analyses, only 5(20\%) interventions reported using intent-to-treat analyses; this in turn may have impacted the positive effect of the intervention across the interventions. The positive effect found in these interventions were only limited to participants who were present for follow-up assessments and did not account for attrition. There were variations in the reporting of attrition rates across the interventions, and few studies provided information on reasons for attrition. Information on the reasons for attrition may help to highlight barriers or challenges that influence AGYW participation in the interventions. For instance, one of the interventions encountered some logistics challenges in the form of delays in providing loans to participants [84]. Such logistical challenges are critical information that may influence AGYW attrition and participation in HIV prevention interventions. Thus, efforts to account for factors influencing attrition are necessary for identifying barriers and challenges to AGYW continued participation in interventions.

For adoption, the description of the intervention location, staff delivering the intervention, and level of staff expertise were well documented in the reviewed interventions. However, there was minimal reporting on the methods used to enhance staff and intervention settings adoption. This is consistent with other reviews using the RE-AIM framework, where there is consistent under-reporting on methods used to enhance adoption by intervention delivery agents [111, 118]. This makes it challenging to determine what types of delivery agents may be appropriate for the optimal implementation of the intervention [111]. Furthermore, only 3(12\%) interventions reported on the intervention adoption rate. Reporting of adoption rate and characteristics of participating intervention locations versus non-participating locations may help highlight components of intervention design that either hinder or foster adoption across various settings [111].

The cost of intervention implementation is an important factor in determining the translation of research findings to real-world settings. Three $(12 \%)$ of the 25 interventions in the review reported on the cost of intervention delivery. The findings of the review reveal a paucity of data on the cost and cost-effectiveness of implementing economic empowerment HIV prevention interventions among AGYW. Report on delivery cost allows for effective planning to optimize the yield and reach of economic empowerment HIV prevention for AGYW [119-121]. Likewise, documenting cost-effectiveness is crucial for sustainability and large-scale dissemination of HIV prevention interventions in SSA [120]. Cost information also helps to allocate resources efficiently particularly in settings were resources are scare. This in turn may help maximize the impact of positive health outcomes among AGYW [119].

In terms of implementation, intervention duration and frequency were consistently measured across the interventions. However, none of the interventions reported on the fidelity of the study, although it is a critical measure of the internal validity of the interventions. Therefore, it is unclear if the reported intervention impact were attributed to the fidelity of the intervention or to the actual intervention components [110]. Considering the critical role of these components in enhancing the impact and scale-up of such intervention, the scarcity of evidence in this area is a concern. Future interventions should clearly specify implementation components such as fidelity to enhance the translation of these interventions to other settings and populations.

Regarding maintenance, about 7(26\%) reported on this RE-AIM dimension. This is a favorable result, compared to other reviews that have reported between $0.0 \%$ and $11.0 \%$ maintenance $[110,122]$. This RE-AIM dimension helps to understand the long-term maintenance of behavior change among intervention participants and the sustainability of the interventions at implementing locations. Most of the interventions measured maintenance of individual behavior at least 6 months following the completion of the intervention, with only 4 of the interventions measuring behavior at 48 months after intervention completion. While individual-level maintenance components were frequently reported, little attention was paid to the assessment of setting- and program-level maintenance components. Intervention maintenance also known as sustainability is influenced by an interplay of individual-, program-level factors and broader sociocultural- and community-level factors, which collectively determine long-term intervention impact. Therefore, future research should address critical gaps in the assessment of intervention maintenance, and apply a more comprehensive approach in the evaluation of this implementation outcome dimension.

\section{Limitations}

Our review has some limitations. First, our conclusions are based on the degree to which the included interventions reported on specific RE-AIM dimensions. It is possible that some of the RE-AIM dimensions were measured, but not reported in the interventions due to editorial restrictions. To address this limitation, we included all available articles on a specific intervention. 
Second, we did not conduct a meta-analysis. While this was not the focus of this systematic review, the heterogeneity of the included interventions and variations in HIV prevention outcomes would not have supported a meta-analysis. Third, our search strategy was limited to published articles and those available in English; this is potentially subject to selection bias. Fourth, it is worth noting that the Cochrane Collaboration risk of bias assessment tool used in assessing study quality is biased towards purely quantitative study designs and quite limited in appraising mixed and qualitative study designs. Given the limitation of this tool, it was only used to evaluate the internal validity of the interventions included in the review and not to select articles included in the review.

Nonetheless, this study has a number of strengths. First, this review was conducted with a well-constructed search strategy, created with the help of the college librarian, and was supplemented by a manual search of the reference list of included articles. Second, to the best of our knowledge, this is the first study to collate and examine the measurement of implementation outcomes among economic empowerment interventions HIV prevention for adolescent girls and young women in subSaharan Africa using the RE-AIM framework as a guide.

\section{Conclusion}

Emerging evidence suggests that economic strengthening interventions can be effective in reducing adolescent girls' and young women's risks for HIV. RE-AIM assessment showed that economic empowerment intervention provides AGYW with skills to reduce their risk of HIV. Our findings further show that although researchers frequently reported on intervention-specific implementation science outcome components, broad or system-level implementation outcome indicators of these interventions are scarce. Considering the critical role of these implementation factors in enhancing the ultimate impact of combination economic strenghtening intervention on HIV prevention among AGYW in SSA, the scarcity of evidence is a concern. We recommend the use of REAIM components in future EE HIV interventions targeting AGYW, with special consideration given to factors relevant to the adoption, implementation (such as implementation cost, adoption rate, and intervention fidelity) and long-term sustainability of these interventions in SSA. We further suggest the measurement of other implementation science outcomes beyond RE-AIM indicators to provide a holistic indicator of factors and measures to promote intervention scale-up, dissemination and sustainability. Overall, the findings of this systematic review and the use of the RE-AIM framework, have the potential to accelerate the tempo of implementation and dissemination of evidence-based interventions for addressing HIV prevention among atrisk AGYW in SSA.

\section{Supplementary information}

Supplementary information accompanies this paper at https://doi.org/10. 1186/s43058-020-00042-4.

Additional file 1. PRISMA checklist.

Additional file 2. Search strategy.

Additional file 3. RE-AIM components extraction.

\section{Abbreviations}

AGYW: Adolescent girls and young women; CCTs: Conditional cash transfer CA: Collins Airhihenbuwa; CO: Chisom Obiezu-Umeh; FE: Fred Ssewamala; FU: Florida Uzoaru; HIV: Human immunodeficiency virus; JC: Jamie Curley; J: Juliet Iwelunmor; JE: John Ehiri; OE: Oliver Ezechi; PRISMA: Preferred Reporting Items for Systematic Reviews and Meta-Analyses;

RCTs: Randomized control trials; RE-AIM: Reach, effectiveness, adoption, implementation, and maintenance; SSA: Sub-Saharan Africa; STIs: Sexually transmitted infections

\section{Acknowledgements}

We would like to acknowledge Donghua Tao, MLIS MS PhD (Assistant Director for Information Services, College for Public Health and Social Justice), the college librarian for her assistance with developing a literature search strategy for this systematic review

\section{Authors' contributions}

II conceived the idea for the systematic review. JI and UN abstracted data from paper. JI and UN wrote the first draft of the paper. CO, FU, JE, JC, OE, CA, and FS critically reviewed the manuscript. All authors read and approved the final manuscript

\section{Funding}

No funding was received to carry out this work.

\section{Availability of data and materials}

Articles included in this systematic review are cited in the reference list.

Ethics approval and consent to participate

Not applicable

Consent for publication

Not applicable

\section{Competing interests}

The authors declare that they have no competing interests

\section{Author details}

${ }^{1}$ College for Public Health and Social Justice, Saint Louis University, Salus Center, 3545 Lafayette Avenue, Saint Louis, MO 63104, USA. ${ }^{2}$ Mel and Enid Zuckerman College of Public Health, University of Arizona, 1295 N Martin Avenue, Tucson, AZ 85724, USA. ${ }^{3}$ Nigerian Institute of Medical Research, 6 Edmund Crescent, Yaba, Lagos State, Nigeria. ${ }^{4}$ School of Public Health, Global Research Against Noncommunicable Diseases, Georgia State University, 140 Decatur Street SE, Atlanta, GA 30303, USA. ${ }^{5}$ Brown School, Washington University in Saint Louis, 1 Brookings Drive, Saint Louis, MO 63130, USA

Received: 25 November 2019 Accepted: 24 May 2020

Published online: 10 June 2020

References

1. Karim QA, Baxter C, Birx D. Prevention of HIV in adolescent girls and young women: key to an AIDS-free generation. J Acquir Immune Defic Syndr. 2017 75:S17-26. 
2. Jewkes R, Morrell R. Gender and sexuality: emerging perspectives from the heterosexual epidemic in South Africa and implications for HIV risk and prevention. J Int AIDS Soc. 2010;13(1):6.

3. Muhanguzi FK. Gender and sexual vulnerability of young women in Africa: experiences of young girls in secondary schools in Uganda. Cult Health Sex. 2011;13(06):713-25.

4. Women and HIV: A Spotlight on adolescent girls and young women [https:// www.unaids.org/sites/default/files/media_asset/2019_women-and-hiv_en.pdf].

5. UNAIDS: Women and HIV: spotlight on adolescent girls and young women. In. Geneva; 2019.

6. Karimli L, Ssewamala FM. Do savings mediate changes in adolescents' future orientation and health-related outcomes? Findings from randomized experiment in Uganda. J Adolesc Health. 2015;57(4):425-32.

7. Fox L, Senbet LW, Simbanegavi W. Youth employment in sub-Saharan Africa: challenges, constraints and opportunities. J African Econ. 2016; 25(suppl_1):i3-i15.

8. Filmer D, Fox L. Youth employment in sub-Saharan Africa: the World Bank; 2014.

9. Blum RW. Youth in sub-Saharan Africa. J Adolesc Health. 2007:41(3):230-8.

10. Chatterji M, Murray $N$, London D, Anglewicz P. The factors influencing transactional sex among young men and women in 12 sub-Saharan African countries. Soc Biol. 2005;52(1-2):56-72.

11. Stroeken K, Remes P, De Koker P, Michielsen K, Van Vossole A, Temmerman M. HIV among out-of-school youth in eastern and southern Africa: a review. AIDS Care. 2012;24(2):186-94.

12. Pettifor AE, Levandowski BA, MacPhail C, Padian NS, Cohen MS, Rees HV. Keep them in school: the importance of education as a protective facto against HIV infection among young south African women. Int J Epidemiol. 2008:37(6):1266-73.

13. Birdthistle I, Schaffnit SB, Kwaro D, Shahmanesh M, Ziraba A, Kabiru CW Phillips-Howard P, Chimbindi N, Ondeng'e K, Gourlay A et al: Evaluating the impact of the DREAMS partnership to reduce HIV incidence among adolescent girls and young women in four settings: a study protocol. BMC Public Health 2018, 18(1):N.PAG-N.PAG.

14. NATIONS U: World Youth Report: youth and the 2030 Agenda for sustainable development 2018.

15. Ssewamala FM. Optimizing the "demographic dividend" in young developing countries: the role of contractual savings and insurance for financing education. Int J Soc Welfare. 2015;24(3):248-62.

16. Ssewamala FM, Sperber $E$, Zimmerman JM, Karimli $L$. The potential of assetbased development strategies for poverty alleviation in sub-Saharan Africa. Int J Soc Welfare. 2010;19(4):433-43.

17. Ross DA, Dick B, Ferguson J, Organization WH: Preventing HIV/AIDS in young people: a systematic review of the evidence from developing countries. World Health Organization 2006.

18. Pettifor A, MacPhail C, Hughes JP, Selin A, Wang J, Gómez-Olivé FX, et al. The effect of a conditional cash transfer on HIV incidence in young women in rural South Africa (HPTN 068): a phase 3, randomised controlled trial. Lancet Glob Health. 2016:4(12):e978-88.

19. Pettifor A, Bekker L-G, Hosek S, DiClemente R, Rosenberg M, Bull S, et al. Preventing HIV among young people: research priorities for the future. $J$ Acquir Immune Defic Syndr (1999). 2013;63(0 2):S155.

20. Rotheram-Borus MJ, Davis E, Rezai R. Stopping the rise of HIV among adolescents globally. Curr Opin Pediatr. 2018;30(1):131-6.

21. Cho H, Hallfors DD, Mbai II, Itindi J, Milimo BW, Halpern CT, et al. Keeping adolescent orphans in school to prevent human immunodeficiency virus infection: evidence from a randomized controlled trial in Kenya. J Adolesc Health. 2011:48(5):523-6.

22. Hallfors D, Cho H, Rusakaniko S, Iritani B, Mapfumo J, Halpern C. Supporting adolescent orphan girls to stay in school as HIV risk prevention: evidence from a randomized controlled trial in Zimbabwe. Am J Public Health. 2011; 101(6):1082-8

23. Hallfors DD, Cho H, Rusakaniko S, Mapfumo J, Iritani B, Zhang L, et al. The impact of school subsidies on HIV-related outcomes among adolescent female orphans. J Adolesc Health. 2015;56(1):79-84.

24. Gallant M, Maticka-Tyndale E. School-based HIV prevention programmes for African youth. Soc Sci Med. 2004;58(7):1337-51.

25. Mantell JE, Harrison A, Hoffman S, Smit JA, Stein ZA, Exner TM. The Mpondombili project: preventing HIV/AIDS and unintended pregnancy among rural south African school-going adolescents. Reprod Health Matters. 2006;14(28):113-22.
26. Ssewamala FM, Ismayilova L, McKay M, Sperber E, Bannon W Jr, Alicea S Gender and the effects of an economic empowerment program on attitudes toward sexual risk-taking among AIDS-orphaned adolescent youth in Uganda. J Adolesc Health. 2010:46(4):372-8.

27. Fiscian VS, Obeng EK, Goldstein K, Shea JA, Turner BJ. Adapting a multifaceted US HIV prevention education program for girls in Ghana. AIDS Educ Prev. 2009;21(1):67-79.

28. Harrison A, Hoffman S, Mantell JE, Smit JA, Leu C-S, Exner TM, et al. Genderfocused HIV and pregnancy prevention for school-going adolescents: the Mpondombili pilot intervention in KwaZulu-Natal, South Africa. J HIV/AIDS Soc Services. 2016:15(1):29-47.

29. Underwood C, Brown J, Schwandt H: Multi-level responses to multi-level vulnerabilities: creating an enabling environment for HIV prevention for girls in Botswana, Malawi, and Mozambique. In: Structural Dynamics of HIV. edn.: Springer; 2018: 115-130.

30. Rosenberg NE, Bhushan NL, Vansia D, Phanga T, Maseko B, Nthani T, et al. Comparing youth-friendly health services to the standard of care through "girl power-Malawi": a quasi-experimental cohort study. J Acquir Immune Defic Syndr. 2018;79(4):458-66.

31. Wingood GM, Reddy P, Lang DL, Saleh-Onoya D, Braxton N, Sifunda S, et al. Efficacy of SISTA South Africa on sexual behavior and relationship control among isiXhosa women in South Africa: results of a randomized-controlled trial. J Acquir Immune Defic Syndr (1999). 2013;63(0 1):S59-65.

32. Medley A, Kennedy C, O'Reilly K, Sweat M. Effectiveness of peer education interventions for HIV prevention in developing countries: a systematic review and meta-analysis. AIDS Educ Prev. 2009;21(3):181-206.

33. Harrison A, Newell M-L, Imrie J, Hoddinott G. HIV prevention for south African youth: which interventions work? A systematic review of current evidence. BMC Public Health. 2010;10(1):102.

34. Michielsen $\mathrm{K}$, Chersich MF, Luchters $\mathrm{S}$, De Koker $\mathrm{P}$, Van Rossem $\mathrm{R}$ Temmerman M. Effectiveness of HIV prevention for youth in sub-Saharan Africa: systematic review and meta-analysis of randomized and nonrandomized trials. AIDS. 2010;24(8):1193-202.

35. Fonner VA, Armstrong KS, Kennedy CE, O'Reilly KR, Sweat MD. School based sex education and HIV prevention in low-and middle-income countries: a systematic review and meta-analysis. PLoS One. 2014;9(3): e89692.

36. Kennedy CE, Fonner VA, O'Reilly KR, Sweat MD. A systematic review of income generation interventions, including microfinance and vocational skills training, for HIV prevention. AIDS Care. 2014;26(6):659-73.

37. Dworkin SL, Blankenship K. Microfinance and HIV/AIDS prevention: assessing its promise and limitations. AIDS Behav. 2009;13(3):462-9.

38. Swann M. Economic strengthening for HIV prevention and risk reduction: a review of the evidence. AIDS Care. 2018;30(sup3):37-84.

39. Ssewamala FM, Ismayilova L. Integrating children's savings accounts in the care and support of orphaned adolescents in rural Uganda. Soc Serv Rev. 2009:83(3):453-72.

40. Hardee K, Gay J, Croce-Galis M, Peltz A. Strengthening the enabling environment for women and girls: what is the evidence in social and structural approaches in the HIV response? J Int AIDS Soc. 2014;17(1):18619.

41. Stark L, Seff I, Assezenew A, Eoomkham J, Falb K, Ssewamala FM. Effects of a social empowerment intervention on economic vulnerability for adolescent refugee girls in Ethiopia. J Adolesc Health. 2018;62(1):S15-20.

42. Padian NS, McCoy SI, Karim SSA, Hasen N, Kim J, Bartos M, et al. HIV prevention transformed: the new prevention research agenda. Lancet. 2011; 378(9787):269-78

43. Unterhalter E. Inequality, capabilities and poverty in four African countries: girls' voice, schooling, and strategies for institutional change. Cambridge J Educ. 2012;42(3):307-25.

44. Masanjala W. The poverty-HIV/AIDS nexus in Africa: a livelihood approach. Soc Sci Med. 2007;64(5):1032-41.

45. Hallman K. Socioeconomic disadvantage and unsafe sexual behaviors among young women and men in South Africa; 2004

46. Greig FE, Koopman C. Multilevel analysis of women's empowerment and HIV prevention: quantitative survey results from a preliminary study in Botswana. AIDS Behav. 2003;7(2):195-208.

47. Bryceson DF, Fonseca J. Risking death for survival: peasant responses to hunger and HIV/AIDS in Malawi. World Dev. 2006;34(9):1654-66.

48. Morris ZS, Wooding S, Grant J. The answer is 17 years, what is the question: understanding time lags in translational research. J Royal Soc Med. 2011 104(12):510-20. 
49. Trochim W: Translation won't happen without dissemination and implementation: Some measurement and evaluation issues. In: 3rd Annual Conference on the Science of Dissemination and Implementation Bethesda, MD: NIH Office of Behavioral and Social Sciences Research: 2010; 2010.

50. Davis D, Davis ME, Jadad A, Perrier L, Rath D, Ryan D, et al. The case for knowledge translation: shortening the journey from evidence to effect. BMJ. 2003;327(7405):33-5

51. Proctor $E$, Silmere $H$, Raghavan $R$, Hovmand $P$, Aarons $G$, Bunger $A$, et al. Outcomes for implementation research: conceptual distinctions, measurement challenges, and research agenda. Adm Policy Ment Health Ment Health Serv Res. 2011;38(2):65-76.

52. Curran GM, Bauer M, Mittman B, Pyne JM, Stetler C. Effectivenessimplementation hybrid designs: combining elements of clinical effectiveness and implementation research to enhance public health impact. Med Care. 2012;50(3):217.

53. Schackman BR. Implementation science for the prevention and treatment of HIV/AIDS. J Acquir Immune Defic Syndr (1999). 2010;55(Suppl 1):S27.

54. Glasgow RE, Eckstein ET, ElZarrad MK. Implementation science perspectives and opportunities for HIV/AIDS research: integrating science, practice, and policy. JAIDS J Acquir Immune Defic Syndr. 2013;63:S26-31.

55. Peters DH, Adam T, Alonge O, Agyepong IA, Tran N. Implementation research: what it is and how to do it. BMJ. 2013;347:f6753.

56. Lobb R, Colditz GA. Implementation science and its application to population health. Annu Rev Public Health. 2013;34:235-51.

57. Dellar RC, Dlamini S, Karim QA. Adolescent girls and young women: key populations for HIV epidemic control. J Int AIDS Soc. 2015;18:19408.

58. Glasgow RE, Lichtenstein E, Marcus AC. Why don't we see more translation of health promotion research to practice? Rethinking the efficacy-toeffectiveness transition. Am J Public Health. 2003;93(8):1261-7.

59. Glasgow RE, Vogt TM, Boles SM. Evaluating the public health impact of health promotion interventions: the RE-AIM framework. Am J Public Health. 1999;89(9):1322-7.

60. Kim J, Pronyk P, Barnett T, Watts C. Exploring the role of economic empowerment in HIV prevention. Aids. 2008;22:S57-71.

61. Buvinić M, Furst-Nichols R. Promoting women's economic empowerment: what works? World Bank Res Obs. 2014;31(1):59-101.

62. Stewart R. Changing the world one systematic review at a time: a new development methodology for making a difference. Dev South Afr. 2014;31(4):581-90.

63. Gaglio B, Shoup JA, Glasgow RE. The RE-AIM framework: a systematic review of use over time. Am J Public Health. 2013;103(6):e38-46.

64. Harden SM, Gaglio B, Shoup JA, Kinney KA, Johnson SB, Brito F, et al. Fidelity to and comparative results across behavioral interventions evaluated through the RE-AIM framework: a systematic review. Syst Rev. 2015;4(1):155.

65. Akers JD, Estabrooks PA, Davy BM. Translational research: bridging the gap between long-term weight loss maintenance research and practice. Journal of the American Dietetic Association 2010. 110(10):1511-1522. e1513.

66. Higgins JP, Altman DG, Gøtzsche PC, Jüni P, Moher D, Oxman AD, et al. The Cochrane Collaboration's tool for assessing risk of bias in randomised trials. BMJ. 2011;343:d5928.

67. Higgins JP, Green S: Cochrane handbook for systematic reviews of interventions, vol. 4: John Wiley \& Sons; 2011. https://training.cochrane.org/ handbook/archive/v5.1/.

68. Abdool Karim Q. Impact of conditional cash incentives on HSV-2 and HIV prevention in rural high school students in South Africa: the CAPRISA 007 cluster randomized controlled trial. In: Vancouver: International AIDS Conference. 2015;2015

69. Humphries $H$, Kharsany AB, Leask K, Ntombela F, Karim QA: The impact of conditional cash transfers in reducing HIV in adolescent girls and boys (RHIVA): the CAPRISA 007 matched pair, cluster randomised controlled trial. In: The CAPRISA Clinical Trials: HIV Treatment and Prevention. edn.: Springer; 2017: 77-89

70. Adoho F, Chakravarty S, Korkoyah DT, Lundberg M, Tasneem A. The impact of an adolescent girls employment program: the EPAG project in Liberia: the World Bank; 2014

71. Austrian K, Muthengi E. Can economic assets increase girls' risk of sexual harassment? Evaluation results from a social, health and economic assetbuilding intervention for vulnerable adolescent girls in Uganda. Child Youth Serv Rev. 2014;47:168-75

72. Muthengi E, Austrian K, Gitau J. Preliminary findings from social and economic assets for vulnerable adolescent girls baseline study. Population Council: Nairobi, Kenya; 2014.
73. Baird SJ, Garfein RS, McIntosh CT, Özler B. Effect of a cash transfer programme for schooling on prevalence of HIV and herpes simplex type 2 in Malawi: a cluster randomised trial. Lancet. 2012;379(9823):1320-9.

74. Baird SJ, Chirwa E, De Hoop J, Özler B: Girl power: cash transfers and adolescent welfare. Evidence from a cluster-randomized experiment in Malawi. In.: National Bureau of Economic Research; 2013.

75. Bandiera O, Buehren N, Burgess R, Goldstein M, Gulesci S, Rasul I, et al. Empowering adolescent girls: evidence from a randomized control trial in Uganda: World Bank; 2012

76. Bandiera O, Buehren N, Burgess R, Goldstein M, Gulesci S, Rasul I, et al. Women's empowerment in action: evidence from a randomized control trial in Africa: World Bank; 2018.

77. Bazika JB: Effectiveness of small scale income generating activities in reducing risk of HIV in youth in the Republic of Congo. AIDS care 2007, 19(sup 1):23-24

78. Cho H, Mbai I, Luseno WK, Hobbs M, Halpern C, Hallfors DD. School support as structural HIV prevention for adolescent orphans in western Kenya. J Adolesc Health. 2018;62(1):44-51.

79. Cluver LD, Orkin MF, Yakubovich AR, Sherr L: Combination social protection for reducing HIV-risk behavior amongst adolescents in South Africa. Journal of acquired immune deficiency syndromes (1999) 2016, 72(1):96.

80. De Walque D, Dow WH, Nathan R, Abdul R, Abilahi F, Gong E, et al. Incentivising safe sex: a randomised trial of conditional cash transfers for HIV and sexually transmitted infection prevention in rural Tanzania. BMJ Open. 2012;2(1):e000747.

81. de Walque D, Dow WH, Nathan R. Rewarding safer sex: conditional cash transfers for HIV/STI prevention: the World Bank; 2014.

82. Dunbar MS, Maternowska MC, Kang MS, Laver SM, Mudekunye-Mahaka I, Padian NS. Findings from SHAZ!: a feasibility study of a microcredit and lifeskills HIV prevention intervention to reduce risk among adolescent female orphans in Zimbabwe. J Prev Interv Community. 2010;38(2):147-61.

83. Dunbar MS, Dufour M-SK, Lambdin B, Mudekunye-Mahaka I, Nhamo D, Padian NS. The SHAZ! Project: results from a pilot randomized trial of a structural intervention to prevent HIV among adolescent women in Zimbabwe. PLoS One. 2014;9(11):e113621.

84. Erulkar A, Chong E. Evaluation of a savings \& micro-credit program for vulnerable young women in Nairobi: Population Council; 2005.

85. Hall J, Dondo A, Sebstad J. Tap and reposition youth (TRY) program: providing social support, savings and microcredit opportunities to adolescent girls at risk for HIV/AIDS in Kenya. Microfinance Brief. 2006.

86. Goodman ML, Kaberia R, Morgan RO, Keiser PH. Health and livelihood outcomes associated with participation in a community-based empowerment program for orphan families in semirural Kenya: a crosssectional study. Vulnerable Children Youth Studies. 2014;9(4):365-76.

87. Luseno W, Zhang L, Rusakaniko S, Cho H, Hallfors D. HIV infection and related risk behaviors: does school support level the playing field between orphans and nonorphans in Zimbabwe? AIDS Care. 2015;27(9): 1191-5.

88. Handa S, Halpern CT, Pettifor A, Thirumurthy $H$. The government of Kenya's cash transfer program reduces the risk of sexual debut among young people age 15-25. PLoS One. 2014;9(1):e85473.

89. Rosenberg M, Pettifor A, Thirumurthy H, Halpern $C T$, Handa S. The impact of a national poverty reduction program on the characteristics of sex partners among Kenyan adolescents. AIDS Behav. 2014;18(2):311-6.

90. Jewkes R, Gibbs A, Jama-Shai N, Willan S, Misselhorn A, Mushinga M, et al. Stepping stones and creating futures intervention: shortened interrupted time series evaluation of a behavioural and structural health promotion and violence prevention intervention for young people in informal settlements in Durban, South Africa. BMC Public Health. 2014;14(1):1325.

91. Khoza N, Stadler J, MacPhail C, Chikandiwa A, Brahmbhatt H, DelanyMoretlwe S. Cash transfer interventions for sexual health: meanings and experiences of adolescent males and females in inner-city Johannesburg. BMC Public Health. 2018;18(1):120.

92. Kim J, Ferrari G, Abramsky T, Watts C, Hargreaves J, Morison L, et al. Assessing the incremental effects of combining economic and health interventions: the IMAGE study in South Africa. Bull World Health Organ. 2009;87:824-32.

93. Pronyk PM, Hargreaves JR, Kim JC, Morison LA, Phetla G, Watts C, et al. Effect of a structural intervention for the prevention of intimate-partner violence and HIV in rural South Africa: a cluster randomised trial. Lancet. 2006;368(9551):1973-83. 
94. Kim JC, Watts CH, Hargreaves JR, Ndhlovu LX, Phetla G, Morison LA, et al. Understanding the impact of a microfinance-based intervention on women's empowerment and the reduction of intimate partner violence in South Africa. Am J Public Health. 2007:97(10):1794-802.

95. Pronyk PM, Kim JC, Abramsky T, Phetla G, Hargreaves JR, Morison LA, et al. A combined microfinance and training intervention can reduce HIV risk behaviour in young female participants. Aids. 2008;22(13):1659-65.

96. Kohler H-P, Thornton RL. Conditional cash transfers and HIV/AIDS prevention: unconditionally promising? World Bank Econ Rev. 2011;26(2):165-90.

97. Nyqvist MB, Corno L, De Walque D, Svensson J. Using lotteries to incentivize safer sexual behavior: evidence from a randomized controlled trial on HIV prevention: the World Bank; 2015.

98. Björkman Nyqvist M, Corno L, De Walque D, Svensson J. Incentivizing safer sexual behavior: evidence from a lottery experiment on HIV prevention. Am Econ J Appl Econ. 2018;10(3):287-314.

99. O’Neill Berry M, Kuriansky J, Lytle MC, Vistman B, Mosisili MS, Hlothoane L, et al. Entrepreneurial training for girls empowerment in Lesotho: a process evaluation of a model programme. S Afr J Psychol. 2013;43(4):445-58.

100. Pettifor A, MacPhail C, Selin A, Gómez-Olivé FX, Rosenberg M, Wagner RG, et al. HPTN 068: a randomized control trial of a conditional cash transfer to reduce HIV infection in young women in South Africa-study design and baseline results. AIDS Behav. 2016;20(9):1863-82.

101. Rotheram-Borus MJ, Lightfoot M, Kasirye R, Desmond K. Vocational training with HIV prevention for Ugandan youth. AIDS Behav. 2012;16(5):1133-7.

102. Ssewamala FM, Han C-K, Neilands TB, Ismayilova L, Sperber E. Effect of economic assets on sexual risk-taking intentions among orphaned adolescents in Uganda. Am J Public Health. 2010;100(3):483-8.

103. Ismayilova L, Ssewamala FM, Karimli L. Family support as a mediator of change in sexual risk-taking attitudes among orphaned adolescents in rural Uganda. J Adolesc Health. 2012;50(3):228-35.

104. Falb K, Tanner S, Asghar K, Souidi S, Mierzwa S, Assazenew A, et al. Implementation of audio-computer assisted self-interview (ACASI) among adolescent girls in humanitarian settings: feasibility, acceptability, and lessons learned. Confl Heal. 2016;10(1):32.

105. Visser M, Zungu N, Ndala-Magoro N. ISIBINDI, creating circles of care for orphans and vulnerable children in South Africa: post-programme outcomes. AIDS Care. 2015;27(8):1014-9.

106. Visser M, Thurman TR, Spyrelis A, Taylor TM, Nice JK, Finestone M. Development and formative evaluation of a family-centred adolescent HIV prevention programme in South Africa. Evaluation and program planning. 2018;68:124-34.

107. Cluver LD, Orkin FM, Boyes ME, Sherr L. Cash plus care: social protection cumulatively mitigates HIV-risk behaviour among adolescents in South Africa. AIDS. 2014;28:S389-97.

108. Jewkes R, Nduna M, Levin J, Jama N, Dunkle K, Puren A, et al. Impact of stepping stones on incidence of HIV and HSV-2 and sexual behaviour in rural South Africa: cluster randomised controlled trial. BMJ. 2008;337:a506.

109. Glasgow RE, Klesges LM, Dzewaltowski DA, Estabrooks PA, Vogt TM. Evaluating the impact of health promotion programs: using the RE-AIM framework to form summary measures for decision making involving complex issues. Health Educ Res. 2006;21(5):688-94.

110. Schlechter CR, Rosenkranz RR, Guagliano JM, Dzewaltowski DA. A systematic review of children's dietary interventions with parents as change agents: application of the RE-AIM framework. Prev Med. 2016;91:233-43.

111. McGoey T, Root Z, Bruner MW, Law B. Evaluation of physical activity interventions in children via the reach, efficacy/effectiveness, adoption, implementation, and maintenance (RE-AIM) framework: a systematic review of randomized and non-randomized trials. Prev Med. 2016;82:8-19.

112. Jang $M$, Chao A, Whittemore R. Evaluating intervention programs targeting parents to manage childhood overweight and obesity: a systematic review using the RE-AIM framework. J Pediatr Nurs. 2015;30(6):877-87.

113. Craike M, Hill B, Gaskin C, Skouteris H. Interventions to improve physical activity during pregnancy: a systematic review on issues of internal and external validity using the RE-AIM framework. BJOG Int J Obstet Gynaecol. 2017;124(4):573-83.

114. Klesges LM, Dzewaltowski DA, Glasgow RE. Review of external validity reporting in childhood obesity prevention research. Am J Prev Med. 2008;34(3):216-23.

115. Blackman KC, Zoellner J, Berrey LM, Alexander R, Fanning J, Hill JL, et al. Assessing the internal and external validity of mobile health physical activity promotion interventions: a systematic literature review using the RE-AIM framework. J Med Internet Res. 2013;15(10):e224.

116. Glasgow RE, Green LW, Ammerman A. A focus on external validity. Evaluation \& the Health Professions. 2007;30(2):115-7.
117. Sarkar S, Corso P, Ebrahim-Zadeh S, Kim P, Charania S, Wall K. Costeffectiveness of HIV prevention interventions in sub-Saharan Africa: a systematic review. EClinicalMedicine. 2019;10:10-31.

118. Zeiler M, Kuso S, Nacke B, Klesges LM, Waldherr K. Evaluating reach, adoption, implementation and maintenance of internet-based interventions to prevent eating disorders in adolescents: a systematic review. Eur J Pub Health. 2019.

119. Cohen DA, Wu S-Y, Farley TA. Comparing the cost-effectiveness of HIV prevention interventions. JAIDS Journal of Acquired Immune Deficiency Syndromes. 2004;37(3):1404-14.

120. Creese A, Floyd K, Alban A, Guinness L. Cost-effectiveness of HIV/AIDS interventions in Africa: a systematic review of the evidence. Lancet. 2002; 359(9318):1635-42.

121. Proctor E, Luke D, Calhoun A, McMillen C, Brownson R, McCrary S, et al. Sustainability of evidence-based healthcare: research agenda, methodological advances, and infrastructure support. Implement Sci. 2015;10(1):88.

122. Allen K, Zoellner J, Motley M, Estabrooks PA: Understanding the internal and external validity of health literacy interventions: a systematic literature review using the RE-AlM framework. Journal of health communication 2011, 16(sup3):55-72.

\section{Publisher's Note}

Springer Nature remains neutral with regard to jurisdictional claims in published maps and institutional affiliations.
Ready to submit your research? Choose BMC and benefit from:

- fast, convenient online submission

- thorough peer review by experienced researchers in your field

- rapid publication on acceptance

- support for research data, including large and complex data types

- gold Open Access which fosters wider collaboration and increased citations

- maximum visibility for your research: over $100 \mathrm{M}$ website views per year

At $\mathrm{BMC}$, research is always in progress.

Learn more biomedcentral.com/submissions 